\title{
Architecture of a multi-crack model with full closing, reopening and sliding capabilities
}

\author{
M. Cervera, G. B. Barbat and M. Chiumenti
}

International Center for Numerical Methods in Engineering (CIMNE)

Technical University of Catalonia - BarcelonaTECH

Edificio C1, Campus Norte, Jordi Girona 1-3

08034 Barcelona, Spain

miguel.cervera@upc.edu,gbarbat@cimne.upc.edu,michele@cimne.upc.edu

\begin{abstract}
This paper discusses the finite element modeling of cracking of quasi-brittle materials under cyclic loading. A damage based crack model is proposed, which considers tensile and compressive damage, irreversible strains upon unloading in compression, as well as micro-crack closure-reopening effects (MCR) with special attention to the sliding of open cracks (MCRS). The model is implemented in conjunction with a mixed strain/displacement finite element formulation which provides enhanced accuracy and guarantees mesh size and bias independent results.

Two distinct damage models are developed. The first one is based on the classical fixed orthotropic crack models and the second is an isotropic version. Both of them incorporate the necessary elements to furnish full MCRS capabilities.

The model is demonstrated through several examples of application and comparison with experimental evidence, in mode I, mode II and mixed mode monotonic and cyclic loading.
\end{abstract}

Keywords: Cracking, Cyclic loading, Damage, Orthotropy, Mixed Finite Elements 


\section{Introduction}

The numerical modelling of tensile cracking in concrete and other quasi brittle materials is a challenging problem that has been an active topic of research for the last 50 years. Springing from the pioneering work of [1] and [2] a broad supply of approaches has been developed for the modeling of cracks in solids. These range from the classical discrete and smeared crack models to the modern phase-field formulations.

In most of the abundant methods, procedures and techniques developed in these 5 decades attention has focused in proportional, monotonic loading and in cracking due to tensile straining. A Rankine-type criterion of admissible stress or strain has been commonly used. The topics of non-proportional, alternate loading and cracking due to shear straining have received far less attention, regardless of their interest in practical situations, e.g. for concrete structures subjected to seismic actions. These topics raise issues such as fixed material systems, stiffness recovery upon load reversal and simultaneous tensile and compressive damage, not present in standard crack models.

Up to date there is no general consensus for the realistic and efficient numerical analysis of failure in quasi-brittle materials [3]. On the contrary, at the same pace as the use of nonlinear numerical analysis became a common use of engineering practice, engineering researchers became growingly aware of the limitations of the ready to use models.

The crack models used in the vast majority of cases, both in practice and research, are those implemented in commercial codes; these are either classical fixed orthotropic models or some damage-based adaptation of those. The bane that this entails arises from two main causes: shear locking and mesh bias dependence. Let us consider them separately, although they are related.

Shear locking was identified as a problem of classical crack models almost from the start. The reasons for shear locking are twofold:

1. From the numerical standpoint, the lack of strain (or stress) continuity in-between adjacent elements in standard irreducible finite elements. In standard FE, the main variable, the displacement field, is approximated and interpolated by means of element-wise defined $C^{0}$ continuous functions, and strains are derived by discrete differentiation within each element from the nodal displacements. This results in an inter-elemental discontinuous discrete strain field and, thru the constitutive equations, in a subsequent inter-elemental discontinuous discrete stress field.

In (linear) problems and isotropic materials, this lack of continuity is frequently used as an error estimator of the numerical solution, as the inter-elemental discontinuity of strain and stress should be reduced on mesh refinement. In problems involving cracking, a severe (nonlinear) orthotropic material behavior is induced, with large differences in the elastic moduli along different directions. In this situation, the discretization error occurring in the evaluation of the strain field is magnified, producing acute stress-locking.

2. From the physical point of view, a fixed orthogonal material system is a poor frame for a nonlinear secant constitutive model in situations in which the principal directions of strain change, e.g. non proportional loading. Material properties and constitutive relations between strain and stress in linear orthotropic materials are defined in fixed orthogonal systems. By analogy, the constitutive behavior of materials with nonlinear induced orthotropy due to cracking are often defined by secant relations in an orthogonal material system which needs to be defined. 
Typically, the orientation of the material axes is defined by the directions of the components of the stress which causes the crack to firstly appear. This presents no difficulty if subsequent loading and unloading of the cracks occurs maintaining the same principal directions, but there are many practical situations in which this does not happen e.g. non proportional loading; loss of co-axiality between strains and stresses aggravates the issue. Under cyclic loading and/or shear dominated situations, these limitations are unwieldy.

The numerical difficulties associated to shear locking in a fixed orthogonal material frame for cracks were soon identified, even for proportional, monotonic loading; the problem was firstly alleviated by the so-called rotating crack models and later by adopting isotropic damage models for tensile failure. The first ones maintained co-axiality of strains and stresses at the cost of rotating the material defects; shear locking was reduced, but not eliminated because of the mentioned lack of inter-element strain continuity in standard FEs. In isotropic damage models, remarkably simple to formulate and implement, shear locking is swiftly eradicated; for this reason, isotropic damage is at the core of phase-field formulations for cracks, despite the obvious locally directional nature of cracks.

The physical limitations of crack models are very clear under non-proportional and alternate loading. For non-proportional loading, the options are either rotating the material axes or allowing for multiple cracks; the first has been discussed above and the second was the motivation for some few multi-crack models $[4,5]$ and the micro-plane theory $[6,7]$. For alternate loading, the features to be incorporated are stiffness recovery for crack closing (microcrack closure-reopening effects, $\mathrm{MCR}$ ) and irrecoverable strains when unloading in compression. It needs to be understood that:

- Rotating crack and isotropic damage models cannot incorporate MCR effects, unless they are provided with some record of the directions in which damage occurred originally.

- In fixed crack models, stiffness recovery upon crack closure leads to unphysical jumps in the shear and transverse stresses if the sliding of the open crack is significant (MCRS effects).

- Irrecoverable strains can be readily incorporated to a crack model, but the formulation adopted may come in conflict with the strain-driven, explicit, secant form of crack models.

As explained, the remedies to the numerical issues raised in certain situations have obvious physical limitations and are often contradictory with the requirements for realistic modeling in some other cases. Reversely, some of the physically motivated requirements are not agreeable from the numerical point of view.

Mesh bias dependence has been a topic of great concern in computational mechanics for the last 30 years, and it has been addressed in a variety of approaches. In linear elasticity, the use of standard FEs cannot guarantee local convergence of the solution in terms of strains and stresses in quasi-singular points such as the re-entrant corners of the tip of a sharp crack. In cracking problems, such situations arise in the vicinity of the tip of propagating cracks, bringing in an incorrect evaluation of the strain and stress in these most critical locations. This lack of local convergence in the strain and stress leads to the spurious mesh bias dependence observed in problems of quasi-brittle crack propagation solved with the standard formulation, yielding incorrect solutions in many cases.

A mixed strain/displacement formulation, proposed and developed in references [8, 9, 10, 11, 12 , 13] resolves the strain independently from the displacement field, instead of being derived from it at element level as in standard FEs. This results in a kinematic enhancement and increase in the accuracy of the strain and stress fields. Seasonably, this enhanced accuracy has proved to eliminate the spurious mesh-dependency and lack of convergence of standard finite elements in 
problems involving isotropic plasticity and damage models without the use of auxiliary tracking techniques and with mesh densities far smaller than those used in phase-field formulations.

In references $[11,12]$ the mixed finite element technology has been assessed for monotonic cracking problems using isotropic and orthotropic damage models in 2D and 3D. Remarkably, the enhancement of the inter-elemental continuity of the strain in mixed FEs alleviates to a large extent the spurious stress locking that used to make orthotropic models unpractical in conjunction with standard FEs. Also, the generality of the mixed finite element formulation allows using it jointly with orthotropic models developed for cyclic loading.

Therefore, the objectives of this paper are: (1) to define the elements of crack models with full MCRS capabilities, (2) to present the formulation of isotropic and orthotropic damage-based models integrating those elements, (3) to demonstrate the performance of the proposed models in situations involving tensile and compressive damage, irreversible strains, MCRS effects and multi-cracking, (4) to demonstrate the capability of the mixed FE formulation in successfully incorporating isotropic and orthotropic damage models subjected to cyclic loading without the numerical burdens associated to standard FEs.

The outline of this paper is as follows. In Section 2 the design requirements of crack models with full MCRS capabilities are given. In Section 3, isotropic and orthotropic fixed d+/d- damagebased crack models are presented; Section 4 incorporates irreversible strains under compression. Section 5 describes the specific treatment of MCR effects; Section 6 incorporates the irreversible strains corresponding to the change of open/closed crack status. Section 7 considers the generation of multi-crack systems for cracking under non-proportional loading. Section 8 demonstrates the performance of the model at local level. Section 9 summarizes the mixed FE formulation. Sections 10 and 11 present numerical simulations of a concrete beam and a metal specimen subjected to cyclic loading where the performance of the constitutive model is examined. Finally, some conclusions are given.

\section{Architecture of crack models with full MCRS capabilities}

The architecture of the multi-crack model with full close-reopening and sliding capabilities in this work consists of the assemblage of the following elements:

1. Strain-driven format.

2. Tensile damage, associated to tensile cracking.

3. Directionality of damage.

4. Irreversibility of damage and its directionality.

5. Secant form.

6. Compressive damage, associated to shear cracking and crushing.

7. Irreversible strains upon compressive unloading.

8. Stiffness recovery upon load reversal, MCR.

9. Stiffness recovery upon load reversal with significant sliding, MCRS.

10. Multi-cracks at local level.

Points 1 to 5 define a classical fixed crack model, and are common to later damage-based orthotropic models. Remarkably, the nowadays very much used isotropic damage models wave crack directionality aside, while rotating orthotropic models abandon its permanent character. Damage is assumed isotropic in phase-field formulations.

Points 6 and 7, relative to the behavior under shear and compression, are present in several orthotropic damage models, based on the split of the tensile/compressive stress or strain tensors 
$[14,15,16,17,18]$, but not applied in fixed material systems. In this work, a completely new approach, based on the activation and deactivation of damage in a fixed material system is adopted.

Note that under shear loading, tensile and compressive stresses act simultaneously in orthogonal directions to each other. This implies that compressive damage needs to be contemplated in shear failure. On the one hand, tensile and compressive damage are not symmetrical; for instance, significant irreversible strains develop upon unloading under compression. On the other hand, compressive damage may be independent from tensile stresses (as in Rankine-type criteria) or related to them (as in Mohr-Coulomb and Drucker-Prager criteria).

Point 8 is necessary for alternate cyclic loading. Previous efforts $[14,15]$ are not applicable to shear dominated situations, unless some record on the directionality of damage at the moment of occurrence is kept, as demonstrated in [17, 18]. Regarding this, in the following, a full record of directionality is maintained, and the ensuing model is therefore "fixed". Stiffness recovery upon load reversal is here toggled through functions that define the status of the cracks as open or closed. The active damage is likewise defined.

Point 9 is closely associated to Point 8; as sliding of the opened cracks occurs, stress continuity needs to be enhanced when the status of cracks, and their stiffness, changes. This is a novel feature of this work; irreversible "sealing" strains are introduced to ensure stress continuity in these situations.

Point 10 is also associated to non-proportional loading and the modification of the directions of straining. This concerned some classical multi-crack models $[4,5]$ and the micro-plane theory $[6$, 7]. Here, a multiplicity of material systems is allowed to develop, and the only one which is active at a current time is defined by the status of the cracks.

The procedures involved in Points $\mathbf{8 , 9}$ and $\mathbf{1 0}$ are simple and effective.

In the following Sections, the different parts of the assemblage are addressed, as well as the algorithms showing how they are implemented and integrated.

\section{Isotropic and orthotropic $\mathrm{d}+/ \mathrm{d}$ - damage model with memory}

In this section, the formulation of the isotropic and orthotropic damage models is presented in secant form. Tensile and compressive damage is considered.

Using Voigt's convention, the strain and stress tensors are expressed as vectors [19]. In 3D the strain vector is $\boldsymbol{\varepsilon}=\left(\varepsilon_{x}, \varepsilon_{y}, \varepsilon_{z}, \gamma_{x y}, \gamma_{x z}, \gamma_{y z}\right)^{T}$ and stress vector is $\boldsymbol{\sigma}=\left(\sigma_{x}, \sigma_{y}, \sigma_{z}, \tau_{x y}, \tau_{x z}, \tau_{y z}\right)^{T}$. In a secant damage model, $\boldsymbol{\varepsilon}$ and $\boldsymbol{\sigma}$ are linked through the constitutive equation:

$$
\boldsymbol{\sigma}=\mathbf{D}(\boldsymbol{d}, \boldsymbol{\theta}) \boldsymbol{\varepsilon}
$$

where $\mathbf{D}=\mathbf{D}(\boldsymbol{d}, \boldsymbol{\theta})$ is the secant constitutive matrix, a function of a set of internal variables $\boldsymbol{d}$ that describe the degradation of the material and $\boldsymbol{\theta}$ which denotes the spatial directions in which damage occurs. Let be $\boldsymbol{d}=\left(d_{1}, d_{2} \cdot d_{3}\right)^{T}$, with the scalar damage indices $d_{i}, i=1,3$, associated to the 3 orthogonal spatial directions of $\boldsymbol{\theta}$.

In materials which are isotropic when undamaged, the orthotropic behavior induced by damage is generally associated to the principal directions of stress (and strain) at the inception of damage. 
In classical "fixed" crack models, the material system $\boldsymbol{\theta}$ was locked thereafter; in "rotating" crack models, the material axes were updated so that stress and strains remain coaxial. Visibly, the differences between both options are the treatment of crack sliding and shear transfer across cracks.

Regardless of the indisputable directional character of cracks, isotropic damage models were firstly proposed [20] and then broadly adopted as crack models as a way of avoiding the numerical issues that orthotropic models bear.

In the following, unless stated otherwise, the material axes $\boldsymbol{\theta}$ will be drawn on as in classical fixed models.

From thermodynamic considerations, the secant matrix needs to be symmetric and positive semidefinite. Symmetric orthotropic damage models may be effectively formulated from the hypothesis of energy equivalence [17, 21, 22, 23].

For an initially isotropy elastic material characterized by the Young's modulus $E$ and the Poisson's ratio $v$, the secant constitutive matrix may be expressed in the $\boldsymbol{\theta}$ material system as

$$
\mathbf{D}=\mathbf{D}(\boldsymbol{d})=
$$

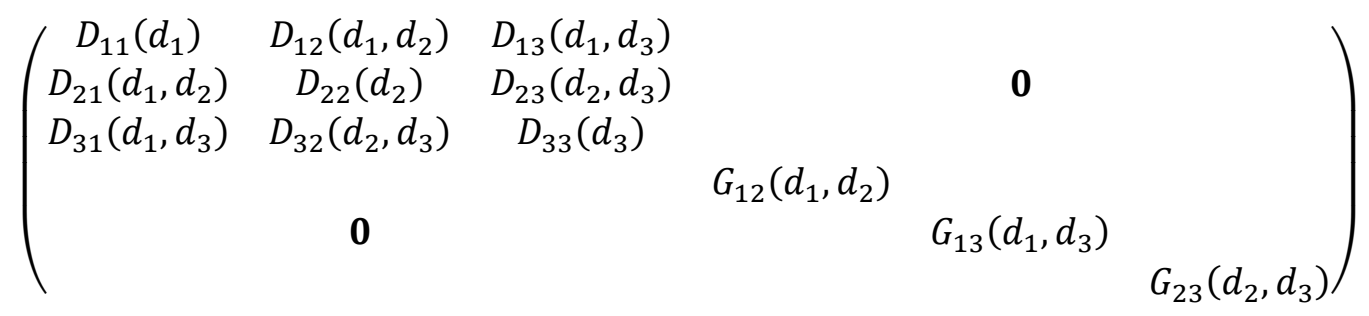

such that

$$
\begin{gathered}
D_{k k}=\left(1-d_{k}\right) D_{k k}^{0} \quad ; \quad k=1,3 \\
D_{k l}=\min \left[\left(1-d_{k}\right) ;\left(1-d_{l}\right)\right] D_{k l}^{0} ; \quad k, l=1,3 \quad k \neq l \\
G_{k l}=\min \left[\left(1-d_{k}\right) ;\left(1-d_{l}\right)\right] G_{k l}^{0} \quad ; \quad k, l=1,3 \quad k \neq l
\end{gathered}
$$

and

$$
\begin{gathered}
D_{k k}^{0}=\frac{E(1-v)}{(1+v)(1-2 v)} \quad ; \quad k=1,3 \\
D_{k l}^{0}=\frac{E v}{(1+v)(1-2 v)} \quad ; \quad k, l=1,3 \quad k \neq l \\
G_{k l}^{0}=\frac{E}{2(1+v)} \quad ; \quad k, l=1,3 \quad k \neq l
\end{gathered}
$$

The isotropic version of the model is obtained by adopting $d_{1}=d_{2}=d_{3}=d$, so that $\mathbf{D}=(1-d) \mathbf{D}_{\mathbf{0}}$, where $d$ is the single scalar internal damage index and $\mathbf{D}_{\mathbf{0}}$ is the elastic constitutive matrix.

The effective stress is defined in terms of the elastic matrix as $\overline{\boldsymbol{\sigma}}=\mathbf{D}_{\mathbf{0}} \boldsymbol{\varepsilon}$. Let $\bar{\sigma}_{i}, i=1,3$, be the three normal stress components of the effective stress $\overline{\boldsymbol{\sigma}}$ in the material axes $\boldsymbol{\theta}$. 
In isotropic models the equivalent effective stress, $\bar{\sigma}_{e q}$, depends on the effective stress $\overline{\boldsymbol{\sigma}}$ through the specific damage criterion $F$

$$
\bar{\sigma}_{e q}=F(\overline{\boldsymbol{\sigma}})
$$

In orthotropic models, an equivalent effective stress is defined for each material direction, $\bar{\sigma}_{e q, i}$, depending on the specific damage criterion $F$ and the material system $\boldsymbol{\theta}$ :

$$
\bar{\sigma}_{e q, i}=F_{i}(\overline{\boldsymbol{\sigma}}, \boldsymbol{\theta}), \quad i=1,3
$$

In this work, Drucker-Prager and Rankine damage criteria are adopted as exemplary cases of isotropic and orthotropic models, respectively. Other criteria may be similarly considered.

The Drucker-Prager criterion depends on the first and second effective stress invariants $I_{1}$ and $J_{2}$ and the equivalent effective stress is defined as

$$
\bar{\sigma}_{e q}=F(\overline{\boldsymbol{\sigma}})=\frac{\left(\sqrt{3 J_{2}}+\alpha I_{1}\right)}{1+\alpha}
$$

with $\alpha$ being a function of the tensile and compressive strengths $f_{t}$ and $f_{c}$

$$
\alpha=\left(\frac{f_{c}}{f_{t}}-1\right) /\left(\frac{f_{c}}{f_{t}}+1\right)
$$

The Rankine criterion depends on the normal effective stresses along the material axes, and the equivalent effective stresses are

$$
\bar{\sigma}_{e q, i}=F_{i}(\overline{\boldsymbol{\sigma}}, \boldsymbol{\theta})=\bar{\sigma}_{i} \quad i=1,3
$$

If tensile and compression damage are to be considered, two distinct failure surfaces $\mathbb{F}_{i}^{ \pm}$need to be explicitly defined, here noted with the + and - suffixes. For an orthotropic model, these surfaces are defined as

$$
\mathbb{F}_{i}^{ \pm}\left(\bar{\sigma}_{e q, i}^{ \pm}, r_{i}^{ \pm}\right)=\bar{\sigma}_{e q, i}^{ \pm}(\overline{\boldsymbol{\sigma}})-r_{i}^{ \pm}=0 \quad i=1,3
$$

where $r_{i}^{+}$and $r_{i}^{-}$are the current tensile and compressive damage thresholds. From the KuhnTucker optimality and consistency conditions, the current values of the damage threshold, defining the actual size of the damage surfaces, are explicitly updated at time $t$ as

$$
r_{i}^{ \pm}=\max \left(r_{0}^{ \pm}, \max \bar{\sigma}_{e q, i}(\hat{t})\right) \quad \hat{t} \in[0, t]
$$

This guarantees the irreversibility of damage and the positiveness of the dissipation. Their initial values depend on the corresponding tensile and compressive uniaxial strengths of the material, $r_{0}^{+}=f^{+}$and $r_{0}^{-}=f^{-}$.

For example, for Rankine's criterion

$$
\bar{\sigma}_{e q, i}^{ \pm}=F_{i}(\overline{\boldsymbol{\sigma}}, \boldsymbol{\theta})=\left\langle \pm \bar{\sigma}_{i}\right\rangle \quad i=1,3
$$

where $\langle\cdot\rangle$ are the Macaulay brackets, such that $\langle x\rangle=x$ if $x \geq 0$, 0 if $x<0$.

The evolution of the internal damage indices may be different for tension and compression in terms of their respective damage threshold internal variables. The requirements on the damage function defining this evolution are: it must be continuous, monotonically increasing from 0 to 1 , and its derivative must be positive. These requirements ensure the continuous and positive 
dissipation of the strain energy and of the mechanical dissipation. In this work, they will follow a parabolic-exponential softening law

$$
d_{i}^{ \pm}=\left\{\begin{array}{lll}
0 & \text { if } & r_{i}^{ \pm}<f_{0}^{ \pm} \\
A_{d}^{ \pm} \frac{f^{ \pm}}{r_{i}^{ \pm}}\left(\frac{r_{i}^{ \pm}-f_{0}^{ \pm}}{f_{p}^{ \pm}-f_{0}^{ \pm}}\right) & \text {if } & f_{0}^{ \pm} \leq r_{i}^{ \pm}<f_{p}^{ \pm} \\
1-\frac{f^{ \pm}}{r_{i}^{ \pm}} \exp \left[2 H_{d}^{ \pm}\left(\frac{f^{ \pm}-r_{i}^{ \pm}}{f^{ \pm}}\right)\right] & \text {if } & r_{i}^{ \pm} \geq f_{p}^{ \pm}
\end{array}\right.
$$

where $f_{0}^{ \pm}=\gamma_{0}^{ \pm} f^{ \pm}$and $f_{p}^{ \pm}=\gamma_{p}^{ \pm} f^{ \pm}$, as well as $\gamma_{0}^{ \pm}$and $\gamma_{p}^{ \pm}$, are material properties. Depending on this,

$$
A_{d}^{ \pm}=\frac{f_{p}^{ \pm}-f^{ \pm}}{f^{ \pm}}
$$

Note that for $\gamma_{0}=\gamma_{p}=1$ the softening law is purely exponential; the parabolic part vanishes.

Energy conservation considerations link the softening parameters, $H_{d}^{+}$to the material fracture energies $G_{f}^{ \pm}$and to the width of the crack $b$ in the following way [3]:

$$
\frac{1}{H_{d}^{ \pm}}=\frac{2 E G_{f}^{ \pm}}{\left(f^{ \pm}\right)^{2}} \frac{1}{b}-\frac{f_{p}^{ \pm}}{f^{ \pm}}-\bar{A}_{\bar{d}}^{ \pm}
$$

with

$$
\bar{A}_{d}^{ \pm}=\frac{A_{d}^{ \pm}\left[\left(f_{p}^{ \pm}\right)^{3}-3 f_{p}^{ \pm}\left(f_{0}^{ \pm}\right)^{2}+2\left(f_{0}^{ \pm}\right)^{3}\right]}{3 f^{ \pm}\left(f_{p}^{ \pm}-f_{0}^{ \pm}\right)^{2}}
$$

The details on the expression of the mechanical dissipation and the derivation of the softening parameters $H_{d}^{ \pm}$can be found in reference [3].

In this work, the bandwidth of the localized cracks is taken as $b=2 h, h$ being the finite element size. This is consistent with the approximation adopted for the discrete strain field in the mixed formulation in Section 9.

For isotropic models, the effective stress is not defined for each material axis. Therefore, the $i$ index is dropped in Eqs. (9), (10), (11), (12) and (13) for the isotropic version of the model. In this way, an isotropic Rankine criterion is defined by taking the maximum principal effective stress as the unique equivalent effective stress

$$
\bar{\sigma}_{e q}^{ \pm}=F(\overline{\boldsymbol{\sigma}})=\left\langle \pm \bar{\sigma}_{1}\right\rangle
$$

For the Drucker-Prager criterion, we will adopt

$$
\bar{\sigma}_{e q}^{ \pm}=F(\overline{\boldsymbol{\sigma}})=\left\langle \pm \frac{\bar{\sigma}_{1}}{\left|\bar{\sigma}_{1}\right|}\right\rangle \frac{\left(\sqrt{3 J_{2}}+\alpha I_{1}\right)}{1+\alpha}
$$

Other alternatives may be contemplated for the assignment of the tensile/compressive equivalent stresses in such a case.

The algorithm for the orthotropic $\mathrm{d}+/ \mathrm{d}$ - crack model is presented in Table 1 . The isotropic version of the model is akin, but there is a unique driving equivalent effective stress $\bar{\sigma}_{e q}$. 
1. Compute the effective stress $\overline{\boldsymbol{\sigma}}=\mathbf{D}_{\mathbf{0}} \boldsymbol{\varepsilon}$

2. IF $\boldsymbol{d}=\mathbf{0}$ THEN

Rotate $\overline{\boldsymbol{\sigma}}$ to its principal directions $\boldsymbol{\theta}$

\section{ELSE}

Rotate $\overline{\boldsymbol{\sigma}}$ to the material axes $\boldsymbol{\theta}$

\section{ENDIF}

3. Compute the equivalent tensile/compressive effective stress $\bar{\sigma}_{\text {eq, } i}^{ \pm} i=1,3$, with Eq. (6)

4. Update the tensile/compressive damage threshold $r_{i}^{ \pm} i=1,3$, with Eq. (11)

5. Update the tensile/compressive damage index $d_{i}^{ \pm} i=1,3$ with Eq. (13)

6. Compute the constitutive matrix $\mathbf{D}(\boldsymbol{d}, \boldsymbol{\theta})$ using Eq. (2)

7. Rotate the secant $\mathbf{D}$ matrix to the global axes

8. Compute $\boldsymbol{\sigma}=\mathbf{D} \varepsilon$ with Eq. (1)

\section{Irreversible strains}

In this section, the orthotropic damage of the precedent Section is extended to incorporate the development of irrecoverable strains upon unloading. This is done by evolving an orthotropic version of the isotropic model used in [24]. Both models are explicitly strain-driven.

In the following, irreversible strains are driven by the compressive strains exclusively; tensile irreversible strains can be readily contemplated.

Let us consider the additive split of the total strain $\boldsymbol{\varepsilon}$ into its elastic $\varepsilon^{e}$ and inelastic (irrecoverable) $\varepsilon^{i}$ parts

$$
\varepsilon=\varepsilon^{e}+\varepsilon^{i}
$$

so that the secant constitutive Eq. (1) is rewritten as

$$
\boldsymbol{\sigma}=\mathbf{D}(\boldsymbol{d}, \boldsymbol{\theta}) \varepsilon^{e}
$$

where $\mathbf{D}(\boldsymbol{d}, \boldsymbol{\theta})$ is the secant damage matrix from the previous Section and $\boldsymbol{\varepsilon}^{e}=\boldsymbol{\varepsilon}-\boldsymbol{\varepsilon}^{i}$. The effective stress is now redefined in terms of the elastic matrix and the elastic strain as $\bar{\sigma}=\mathbf{D}_{\mathbf{0}} \varepsilon^{e}$.

From the 3 directions of the material system $\boldsymbol{\theta}, i=1,3$, direction $k$ is selected, such that

$$
r_{k}^{-}=\max _{i=1,3}\left(r_{i}^{-}\right)
$$

The rate of (compression) irreversible strains is proportional to

- the current elastic strain and

- the increase of the distance to the (compression) damage surface (normalized with respect $r$, the actual size of the (compression) damage surface).

It is stated as:

$$
\dot{\boldsymbol{\varepsilon}}^{i}=\frac{\beta}{1-\beta}\left[\frac{\dot{r}}{r}\right]_{k}^{-} \boldsymbol{\varepsilon}^{e}
$$


where $\beta, 0 \leq \beta \leq 1$, is a material parameter that controls the development of irreversible strains. The super and sub-indices $[\cdot]_{k}^{-}$indicate both the directional and the compressive nature of the argument. Note that the argument cannot be negative because the threshold $r$ may not decrease, Eq. (11). This ensures positive dissipation when irreversible strains develop.

Note that in the limit case $\beta=0$, no irreversible strains develop in the model. Contrariwise, for the limit case $\beta \rightarrow 1$, there is no evolution of the damage threshold $r_{k}^{-}$; in this case, the straining is fully irreversible.

The development of irreversible strain is dissipative. Under the assumption that the total dissipation of the damage model is not affected by the value of parameter $\beta$ the softening modulus $H_{d}^{-}$is redefined as

$$
\frac{1}{H_{d}^{-}}=(1-\beta)\left(\frac{2 E G_{f}^{-}}{\left(f^{-}\right)^{2}} \frac{1}{b}-\frac{f_{p}^{-}}{f^{-}}-\bar{A}_{d}^{-}-\frac{\beta}{1-\beta} B_{d}^{-}\right)
$$

where

$$
B_{d}^{-}=\frac{\left(f_{p}^{-}-f_{0}^{-}\right)\left(3 f_{p}^{-}-2 f^{-} \bar{A}_{d}^{-}+3 f_{0}^{-}\right)}{3\left(f^{-}\right)^{2}}
$$

The supplementary details on the influence of the parameter $\beta$ in the mechanical dissipation and the resulting renewed derivation of the softening parameters $H_{d}^{ \pm}$can be also found in reference [3].

The rate expression for the irreversible strain Eq. (22) may be integrated in time with an implicit backward Euler scheme. Let $\Delta t=t_{n+1}-t_{n}$ be a time increment between subsequent time steps $n$ and $n+1$. In the following, let $\Delta(\cdot)$ indicate the increment of a specific quantity from step $n$ to $n+1$.

The trial effective stresses at $t_{n+1}$ are defined as the effective stresses that would occur without further development of irrecoverable strains,

$$
\overline{\boldsymbol{\sigma}}_{n+1}^{t r}=\left(\left.\overline{\boldsymbol{\sigma}}\right|_{\Delta \varepsilon^{i}=0}\right)_{n+1}=\overline{\boldsymbol{\sigma}}_{n}+\mathbf{D}_{0} \Delta \boldsymbol{\varepsilon}
$$

With some manipulation, it can be shown that

$$
\Delta \boldsymbol{\varepsilon}^{i}=\frac{\beta}{1-\beta}\left[\frac{\Delta r}{r_{n+1}}\right]_{k}^{-} \boldsymbol{\varepsilon}_{n+1}^{e}=\frac{1-\lambda}{\lambda} \boldsymbol{\varepsilon}_{n+1}^{e}
$$

with the scaling parameter $\lambda$

$$
\lambda=1-\beta\left(1-\left[\frac{r_{n}}{r_{n+1}^{t r}}\right]_{k}^{-}\right)
$$

where $\left[r_{n+1}^{t r}\right]_{k}^{-}$is evaluated with $\overline{\boldsymbol{\sigma}}_{n+1}^{t r}$. Note in Eq. (21) that the material parameter $\beta$ defines the rate of the irreversible deformation by scaling the current increment of the equivalent stresses, and consequently the stress threshold. Therefore

$$
[\Delta r]_{k}^{-}=(1-\beta)\left(\left[r_{n+1}^{t r}\right]_{k}^{-}-\left[r_{n}\right]_{k}^{-}\right)
$$

Due to the co-axiality of $\boldsymbol{\varepsilon}^{e}$ and $\Delta \boldsymbol{\varepsilon}^{i}$, it can be shown that $\overline{\boldsymbol{\sigma}}_{n+1}$ are proportional to $\overline{\boldsymbol{\sigma}}_{n+1}^{t r}$, so that

$$
\overline{\boldsymbol{\sigma}}_{n+1}=\lambda \overline{\boldsymbol{\sigma}}_{n+1}^{t r}
$$


This allows taking into account the effect of the irreversible strains explicitly in the computation of the effective stresses prior to the update of the damage indices. The complete algorithm for updating stresses and internal variables is given in Table 2 . Note that it is fully strain-driven.

Table 2. Algorithm for the evaluation of irreversible strains

1. Compute the increment of total strains $\Delta \boldsymbol{\varepsilon}=\boldsymbol{\varepsilon}_{n+1}-\boldsymbol{\varepsilon}_{n}$

2. Compute the trial effective stresses $\overline{\boldsymbol{\sigma}}_{n+1}^{t r}$ with Eq. (25)

3. Select direction $k$ according to Eq. (21)

4. Compute $\left[r_{n+1}^{t r}\right]_{k}^{-}$from Eq. (11) and $\lambda$ from Eq. (27)

5. Compute the effective stress $\overline{\boldsymbol{\sigma}}_{n+1}$ from Eq. (29)

6. Compute $\Delta \varepsilon^{i}$ from Eq. (26)

7. Update $\varepsilon_{n+1}^{i}$ and $\boldsymbol{\varepsilon}_{n+1}^{e}$

8. Compute damage variables $\boldsymbol{d}$ and secant matrix $\mathbf{D}_{n+1}$ according to Table 1

9. Compute $\boldsymbol{\sigma}_{n+1}=\mathbf{D}_{n+1} \boldsymbol{\varepsilon}_{n+1}^{e}$ with Eq. (20)

\section{Microcrack closure-reopening effects (MCR)}

Cyclic straining requires the partial or total recovery of stiffness caused by crack closure and reopening to be resolved. For establishing the status of a crack as active (open) or inactive (closed), a set of step functions is associated to the tensile damage indices. In the orthotropic model, these functions are noted as $H_{i}^{+}$and are defined for each of the directions $i=1,3$, of the material axes $\boldsymbol{\theta}$ as

$$
H_{i}^{+}=\left\{\begin{array}{cc}
0 & \text { if } \bar{\sigma}_{i}=0 \\
\left\langle\frac{\bar{\sigma}_{i}}{\left|\bar{\sigma}_{i}\right|}\right\rangle & \text { if } \bar{\sigma}_{i} \neq 0
\end{array}\right.
$$

where $\bar{\sigma}_{i}$ are the effective normal stresses along direction $i$. Therefore, the crack in direction $i$ is open if $H_{i}^{+}=1$ and it is closed if $H_{i}^{+}=0$.

Associated to the $H_{i}^{+}$functions that signal the current status of the crack in direction $i$, the discrete toggle functions $\mathcal{T}_{i}$ are defined as

$$
\mathcal{T}_{i}= \begin{cases}0 & \text { if } \Delta H_{i}^{+}=0 \\ 1 & \text { if } \Delta H_{i}^{+} \neq 0\end{cases}
$$

where $\Delta H_{i}^{+}$is the increment of $H_{i}^{+}$in the current time (or load) step.

The toggle functions signal if the status of the crack has changed, themselves activating the MCRS effects described in the next Section.

If compressive damage is also considered, an additional set of step functions $H_{i}^{-}$is defined as

$$
H_{i}^{-}= \begin{cases}0 & \text { if } \bar{\sigma}_{i}=0 \\ 1-H_{i}^{+} & \text {if } \bar{\sigma}_{i} \neq 0\end{cases}
$$

The $H_{i}^{+}$and $H_{i}^{-}$step functions are introduced to activate and deactivate the tensile and compressive damage indices in the secant constitutive matrix in each direction $i$. Note that $H_{i}^{+}$ and $H_{i}^{-}$are not equal to one concurrently; hence, tensile and compression damage are not active 
at the same time. The switch between tensile and compressive situations provides partial or total stiffness recovery when the corresponding effective stress flips sign.

Specifically, the active damage $d_{i}$ used in Eq. (3) is posed as

$$
d_{i}=H_{i}^{+} d_{i}^{+}+H_{i}^{-} d_{i}^{-}
$$

In the isotropic model it is also necessary to determine if the tensile or compressive damage indices are active if MCR effects are to be elucidated. To this end, the material axes $\boldsymbol{\theta}$ are associated to the principal directions of stress (and strain) at the inception of damage and they are locked thereafter. The required step and toggle functions are defined by the first material axis, as it is the first to damage, so that

$$
H^{+}=H_{1}^{+} \quad H^{-}=H_{1}^{-} \quad \mathcal{T}=\mathcal{T}_{1}
$$

Consequently, the active damage $d$ is either $d=d^{+}$if the crack is open or $d=d^{-}$if it is closed:

$$
d=H^{+} d^{+}+H^{-} d^{-}
$$

In $3 \mathrm{D}$, the orthotropic model needs to consider 3 damage variables in traction (one for each material direction) and 3 additional damage variables in compression. For isotropic damage, only 2 damage variables, one for tension and one for compression, are needed.

\section{Microcrack closure-reopening effects with sliding (MCRS)}

One of the serious drawbacks of classical fixed crack models is the transfer of shear stress due to sliding of the open cracks. This issue led firstly to the conception of the rotating crack models, which preserved co-axiality of strains and stress at all time at the expense of not fixing the direction of the material cracks, and secondly, to the adoption of isotropic damage models, which further enhanced co-axiality and disavowed directionality.

A secondary drawback of orthogonal crack models, fixed and rotating, was the evolution of Poisson's effect, which was usually discarded from the inception of cracking.

In any case, changes in the secant stiffness lead to discontinuities in the evolution of the stress under continuous straining, an objectionable feature for a constitutive model, burdensome in the numerical application. Note that isotropic damage models treat this issue in a remarkably straightforward manner, one of the reasons for their broad adoption.

These concerns have a predominant role if cyclic straining with stiffness recovery from crack closure-reopening and sliding (MCRS) is considered. Changes in the secant stiffness due to the activation/deactivation of tensile/compressive damage in the material axes $\boldsymbol{\theta}$ are triggered by the individual normal effective stresses $\bar{\sigma}_{i}, i=1,2,3$, but, once implemented, they interact both with the shear strains and with the normal strains in the other directions. If the stress field is to remain continuous in time under continuous straining, "sealing" irrecoverable strains need to be considered; they are associated to the shear and the transverse elastic strains that are present when damage flips occur. In the following, these strains are motivated and evaluated in correspondence with the previous Sections.

Let us consider the secant constitutive Eq. (20)

$$
\boldsymbol{\sigma}=\mathbf{D} \varepsilon^{e}
$$

Differentiating with respect to time 


$$
\dot{\boldsymbol{\sigma}}=\mathbf{D} \dot{\boldsymbol{\varepsilon}}^{e}+\dot{\mathbf{D}} \boldsymbol{\varepsilon}^{e}
$$

where the first term of the right hand side is due to the variation of the total and irrecoverable strains and the second term is due to the variation of the stiffness. In a continuous process in time, continuous strain rates drive continuous stiffness and stress rates. However, changes in stiffness associated to the closure-reopening of the cracks are not strain-drive, but motivated by the change in the open/closed status of the crack, signaled by the toggle functions $\mathcal{T}_{i}$. This means that if stress is to be continuous in time while a change of crack status occurs, there are irreversible strains that must take place concurrently. As in previous Sections, let $n$ and $n+1$ be subsequent time steps, such that the closure or reopening of a crack takes place in the time increment in between. Let $\Delta(\cdot)$ indicate the increment of a specific quantity from step $n$ to $n+1$, and $\Delta t=t_{n+1}-t_{n}$ be the time increment.

Making $\dot{\boldsymbol{\sigma}}=\mathbf{0}$ in Eq. (37) to ensure stress continuity regardless of the variation of stiffness, the incremental irreversible strains caused by crack closure are:

$$
\Delta \boldsymbol{\varepsilon}^{i}=\mathcal{T} \mathbf{D}_{n+1}^{-1} \Delta \mathbf{D} \boldsymbol{\varepsilon}_{n}^{e}
$$

where $\mathcal{T}=\max _{i=1,3}\left(\mathcal{T}_{i}\right) . \Delta \mathbf{D}=\mathbf{D}_{n+1}-\mathbf{D}_{n}$ corresponds to the variation of stiffness due to a change of the open/closed status of the crack. These additional irrecoverable strains guarantee stress continuity at $t_{n}$ and are computed at $t_{n+1}$, added to those computed from Eq. (26).

The algorithm for the orthotropic crack model with irreversible strains and MCRS effects is given in Table 3. The isotropic version of the model is analogous, but there is a unique driving equivalent effective stress $\bar{\sigma}_{e q}$.

Table 3. Algorithm for the orthotropic crack model with irreversible strains and MCRS effects

1. Compute the effective stress $\overline{\boldsymbol{\sigma}}^{\text {tr }}$ with Eq. (25)

2. IF $\boldsymbol{d}=\mathbf{0}$ THEN

Rotate $\overline{\boldsymbol{\sigma}}^{\text {tr }}$ to its principal directions $\boldsymbol{\theta}$

ELSE

Rotate $\overline{\boldsymbol{\sigma}}^{\text {tr }}$ to the material axes $\boldsymbol{\theta}$

ENDIF

3. Compute $\overline{\boldsymbol{\sigma}}$ according to Eq. (29)

4. Compute irreversible strains $\boldsymbol{\varepsilon}^{i}$ following Table 2

5. Compute the equivalent tensile/compressive effective stress $\bar{\sigma}_{e q, i}^{ \pm} \quad i=1,3$, with Eq. (6)

6. Update the tensile/compressive damage threshold $r_{i}^{ \pm} \quad i=1,3$, with Eq. (11)

7. Update the tensile/compressive damage index $d_{i}^{ \pm} i=1,3$, with Eq. (13)

8. Compute the status and toggle functions $H_{i}^{ \pm}, \mathcal{T}_{i} \quad i=1,3$, with Eqs. (30), (31) and (32)

9. Decide on active damage $d$, as in Eq. (33)

10. Compute the constitutive matrix $\mathbf{D}(\boldsymbol{d}, \boldsymbol{\theta})$ using Eq. (2)

11. Decide on changes on crack status $\mathcal{T}=\max _{i=1,3}\left(\mathcal{T}_{i}\right)$

12. If crack status has changed, $\mathcal{T} \neq 0$, compute $\Delta \boldsymbol{\varepsilon}^{i}$ with Eq. (38)

13. Rotate the secant matrix $\mathbf{D}$ and irreversible strains $\boldsymbol{\varepsilon}^{i}$ to the global axes

14. Compute stress $\boldsymbol{\sigma}=\mathbf{D} \boldsymbol{\varepsilon}^{e}$ with Eq. (20) 


\section{Multi-crack model}

Non-proportional loading may produce significant changes in the directions of the principal strains and this may cause multiple cracks in non-orthogonal directions. In cycling situations with pre-loading, these changes occur, inducing cyclic sliding and shearing in the existing cracks.

Fixed orthogonal damage models cannot contemplate the issue of non-orthogonal cracking locally. Rotating and isotropic damage models are even less apt, as they cannot detect so much as the swapping of the principal strain directions under load reversal that occurs under alternate pure shear loading. This concerned some classical multi-crack models $[4,5]$ and the micro-plane theory $[6,7]$.

The activation/deactivation scheme of tensile/compressive damage described in Section 5 allows the consideration of multi-cracks quite naturally. The proceedings are described in the following.

Let the secant constitutive Eq. (20) be

$$
\boldsymbol{\sigma}=\mathbf{D}\left(\boldsymbol{d}^{I}, \boldsymbol{\theta}^{I}\right) \varepsilon^{e}
$$

where $\boldsymbol{d}^{\boldsymbol{I}}$ is the set of damage parameters in the active material system $\boldsymbol{\theta}^{\boldsymbol{I}}$. Note that only one set of material axes can be active at a given time.

Two questions need to be resolved to complete the multi-crack model:

1. The selection of the active material system $\boldsymbol{I}$,

2. The creation of a new material system.

Let $\boldsymbol{J}=\mathbf{1}, \boldsymbol{N}$ be the number of standing material systems. For the selection of the active system $\boldsymbol{I}$, the step functions $\left[H_{1}^{+}\right]^{J}$ are evaluated. The active system $\boldsymbol{I}$ is the one such that

$$
\left[H_{1}^{+} d_{1}^{+}\right]^{I}=\max _{J}\left[H_{1}^{+} d_{1}^{+}\right]^{J}
$$

Systems are discarded if $\left[H_{1}^{+}\right]^{J}=0$ because the corresponding crack is "closed".

A new material system $\boldsymbol{\theta}^{\boldsymbol{N}+\mathbf{1}}$ may be considered if: (a) none of the existing ones is active, and (b) the maximum number of systems allowed is not reached. In this case, the new material system $\boldsymbol{\theta}^{\boldsymbol{N}+1}$, coincides with the current principal directions of effective stress. The new system is locked once it becomes active because damage occurs in it.

In the proposed model the choice of the active crack system depends on tensile damage. If only compressive damage develops because of crushing, according to the criterion in Eq. (40), the activation of a second system does not take place. In such case, in the only existing system compressive damage is active if $H_{i}^{-}=1$; otherwise elastic stiffness is recovered.

For the isotropic version of the multi-crack model, the procedure is analogous, the active system I is $\left[H^{+} d^{+}\right]^{I}=\max _{J}\left[H^{+} d^{+}\right]^{J}$.

The algorithm for the orthotropic multi-crack model is given in Table 4.

As an explanatory remark, note that for alternate shear cyclic loading with an initial precompression (or pre-tension) in 2D, two material systems $\boldsymbol{\theta}^{\mathbf{1}}$ and $\boldsymbol{\theta}^{\mathbf{2}}$ are required. Their relative angles depend on the pre-loading as this determines how much the directions of the principal strains swing from the $90^{\circ}$ corresponding to the pure shear case. The $\boldsymbol{\theta}^{\mathbf{2}}$ system is activated by the alternate tension when system $\boldsymbol{\theta}^{\mathbf{1}}$ is deactivated because axis $x^{1}$ is under compression. 
1. Compute the effective stress $\overline{\boldsymbol{\sigma}}^{t r}$ with Eq. (25)

2. DO for each of the standing material systems $\boldsymbol{J}=\mathbf{1}, \boldsymbol{N}$

Perform steps 2. to 8. From Table 3 ENDDO

3. Select the active material system $\boldsymbol{\theta}^{\boldsymbol{I}}$ according to criterion (40)

4. If no system is active, check for the creation of a new material system $\boldsymbol{\theta}^{\boldsymbol{N}+\mathbf{1}}$

5. Check if system $\boldsymbol{\theta}^{\boldsymbol{N}+\mathbf{1}}$ is active, steps $\mathbf{2}$. To $\mathbf{8}$. From Table 3

6. Compute the constitutive matrix $\mathbf{D}\left(\boldsymbol{d}^{\boldsymbol{I}}, \boldsymbol{\theta}^{\boldsymbol{I}}\right)$ using Eq. (2)

7. Rotate the secant matrix $\mathbf{D}$ and irreversible strains $\boldsymbol{\varepsilon}^{i}$ to the global axes

8. Compute stress $\boldsymbol{\sigma}=\mathbf{D} \boldsymbol{\varepsilon}^{e}$ with Eq. (20)

\section{Study of the model performance}

The behavior of the proposed crack models is first assessed through several cyclic loading numerical tests. The performance of both the isotropic and the orthotropic models is examined. The Rankine criterion is adopted in all the cases. The material properties are shown in Table 5.

\begin{tabular}{|c|c|}
\hline Young's Modulus & $1.5 \cdot 10^{9} \mathrm{~Pa}$ \\
\hline Poisson's Ratio & 0.0 \\
\hline Tensile Strength & $1.0 \cdot 10^{6} \mathrm{~Pa}$ \\
\hline Tensile Fracture Energy & $250 \mathrm{~J} / \mathrm{m}^{2}$ \\
\hline Compressive Strength & $3.0 \cdot 10^{6} \mathrm{~Pa}$ \\
\hline Compressive Fracture Energy & $500 \mathrm{~J} / \mathrm{m}^{2}$ \\
\hline$\gamma_{0}^{+}$parameter & 1.0 \\
\hline$\gamma_{0}^{-}$parameter & 0.5 \\
\hline$\gamma_{p}^{+}$parameter & 1.0 \\
\hline$\gamma_{p}^{-}$parameter & 1.5 \\
\hline$\beta$ parameter for Irreversible Strains & $0.0 / 0.2$ \\
\hline
\end{tabular}

Table 5. Material parameters of the tests

In the tests, the loading is applied via strain increments. Firstly, a vertical pre-tensile or precompressive strain is applied; secondly four different cycles of shear loading with increasing amplitude are executed. For comparison purposes, the test is performed under three different values of initial vertical strain, noted as $\varepsilon_{v}$ :

(a) Pure shear, $\varepsilon_{v}=0.0$

(b) Pre-tension, $\varepsilon_{v}=2.5 \cdot 10^{-4}$

(c) Pre-compression, $\varepsilon_{v}=7.5 \cdot 10^{-4}$

In all the tests, four consecutive cycles of shear that have an amplitude of $\gamma_{x y}=5.0 \cdot 10^{-3}, \gamma_{x y}=$ $1.0 \cdot 10^{-2}, \gamma_{x y}=1.5 \cdot 10^{-2}$ and $\gamma_{x y}=2.0 \cdot 10^{-2}$, respectively, are imposed.

The $\sigma_{x y}-\gamma_{x y}$ curves obtained with the proposed isotropic model with each case are depicted in Figure 1 using a parameter for irreversible strains $\beta=0.0$ (left) and $\beta=0.2$ (right). 

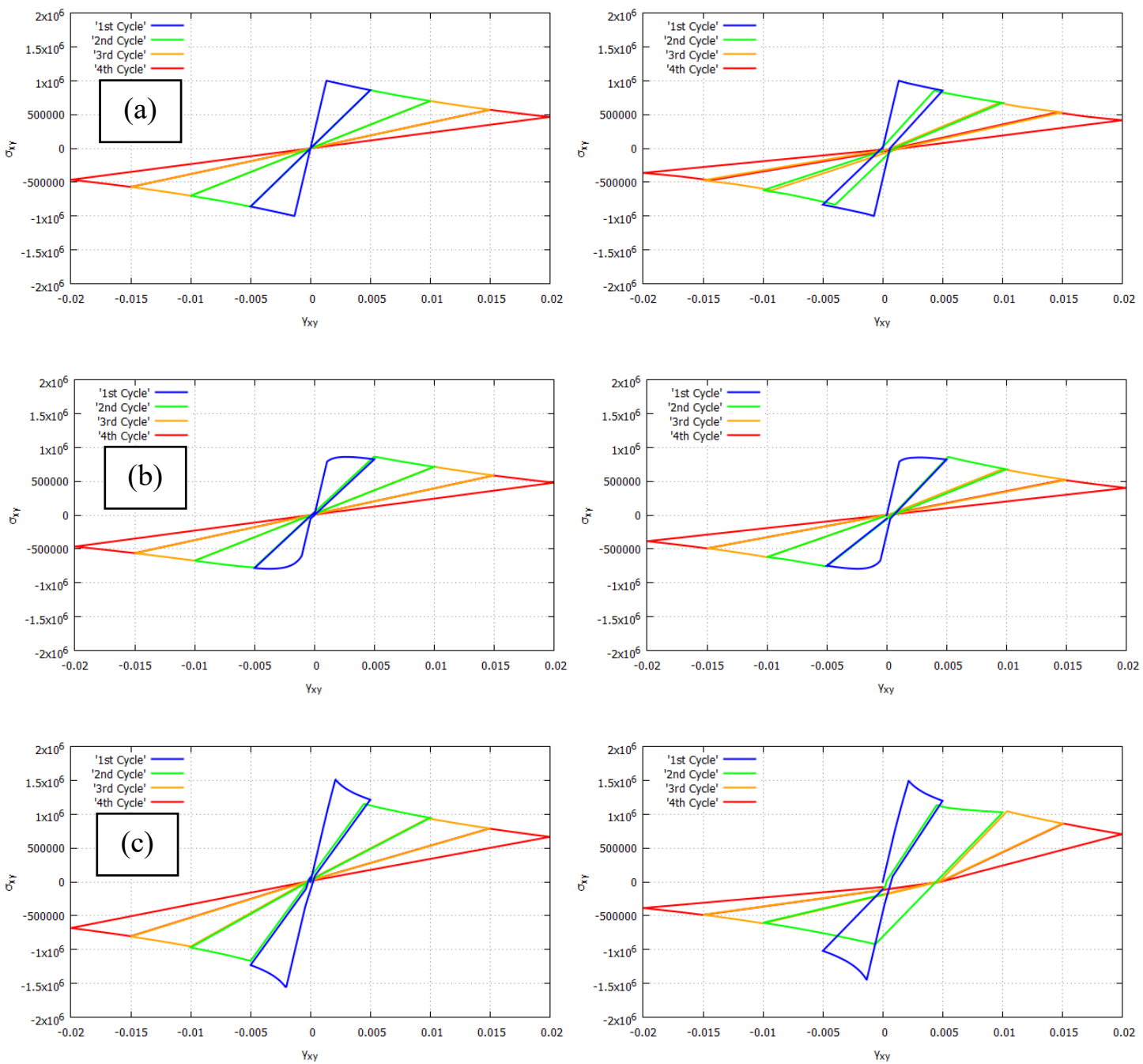

Figure 1. Stress (Pa) vs strain curves of the tests with (a) pure shear, (b) pre-tension and (c) pre-compression with the proposed isotropic model using $\beta=0$ (left) and $\beta=0.2$ (right)

In all cases the stiffness is recovered properly during the cyclic process. Damage develops also when the load is reversed due to consideration in the model of multi-cracks as described in Section 7. For cases (a), proportional loading, pure shear, the principal strains swap directions, and the two cracks formed are orthogonal to each other. However, for cases (b), non-proportional loading, pre-tension, 2 crack systems are necessary, forming angles of $76.91^{\circ}$ and $78.12^{\circ}$ for $\beta=$ $0.0,0.2$, respectively; for cases (c), non-proportional loading, pre-compression, the 2 systems form angles of $61.36^{\circ}$ and $62.77^{\circ}$ for $\beta=0.0,0.2$, respectively. Note that these values differ sensibly from what can be accomplished with a single orthogonal system.

The effect of the non-proportional pre-straining is clear comparing the three cases in the two columns. When pre-tension is applied, the peak shear stress is smaller than in pure shear; the opposite occurs with pre-compression. The first cycle of straining is most affected by the preload, but differences can be observed for all cycles. For $\beta=0.2$, the differences between the three cases are very obvious, because the very asymmetric effect of the compressive irreversible strains is reduced in case (b) and enhanced in case (c). 

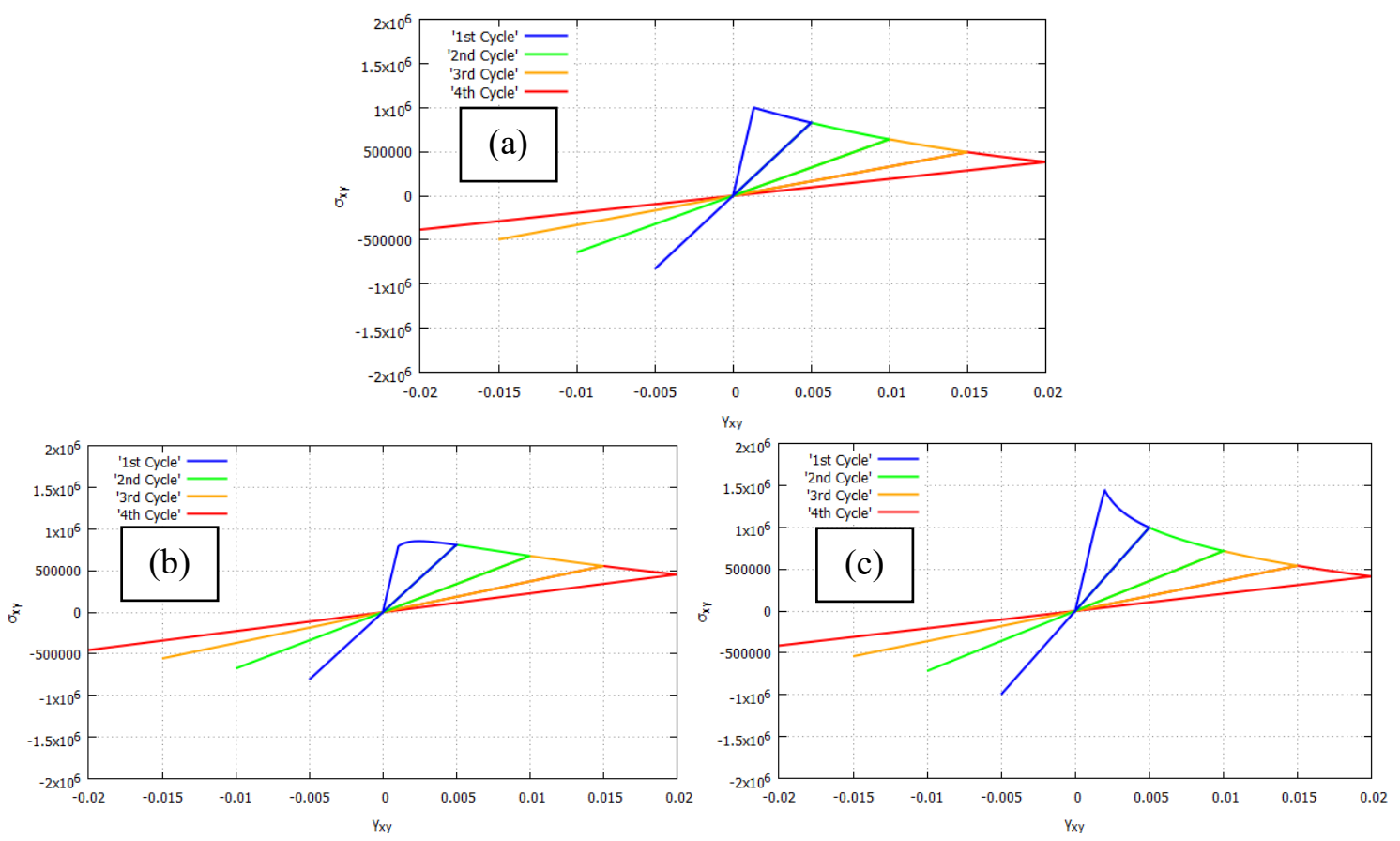

Figure 2. Stress (Pa) vs strain curves of the tests with (a) pure shear, (b) pre-tension and (c) pre-compression with a classical isotropic model

"Sealing" irreversible strains are considered according to the specifications of Section 6, even for the case $\beta=0$, to guarantee the continuity of the stresses when cracks open and close.

Results can also be compared with the ones shown in Figure 2, where a standard (memoryless) isotropic model is used, which cannot reproduce either stiffness recovery or multi-cracking because it has no memory of the directions in which damage originated.

The $\sigma_{x y}-\gamma_{x y}$ curves obtained with the proposed orthotropic model with each loading case are depicted in Figure 3 using a parameter for irreversible strains $\beta=0.0$ (left) and $\beta=0.2$ (right). Proper stiffness recovery takes place when cyclic loading is applied. For all cases, the two material systems form the same in-between angles as for the isotropic model, (a) $90^{\circ}$, (b) $76.91^{\circ}$ and $78.12^{\circ}$ ,(c) $61.39^{\circ}$ and $62.77^{\circ}$ for $\beta=0.0,0.2$, respectively, as these values depend on the effective stresses and, therefore, on the applied straining. The departure from the $90^{\circ}$ of a single material system is significant.

The effects of the pre-tension are opposite to those of the pre-compression, even more seemingly than for the isotropic model, and further evidenced by the asymmetry of the compressive irreversible strains.

Continuity of stresses is enforced at all times; the influence of the sealing strains is most noticeable in the first cycle. See that sealing strains of case (b) in Figure 3 are nearly canceled out by the irreversible strains.

In Figure 4 results corresponding to a classical rotating model are shown; there is no stiffness recovery, because the model has no record of the directions in which damage originated. Consequently, only a minor difference among the three cases is observable.

Figure 5 shows the resulting $\sigma_{x y}-\gamma_{x y}$ curves in the pre-compression case when the sealing irreversible strains, detailed in Section 6, guaranteeing the continuity of the stresses are not considered. It is shown that in this situation the continuity of the stresses is lost when cracks open or close and the stiffness changes accordingly. These results must be compared to the ones in Figure 1c and Figure 3c, where the stress continuity is enforced. 

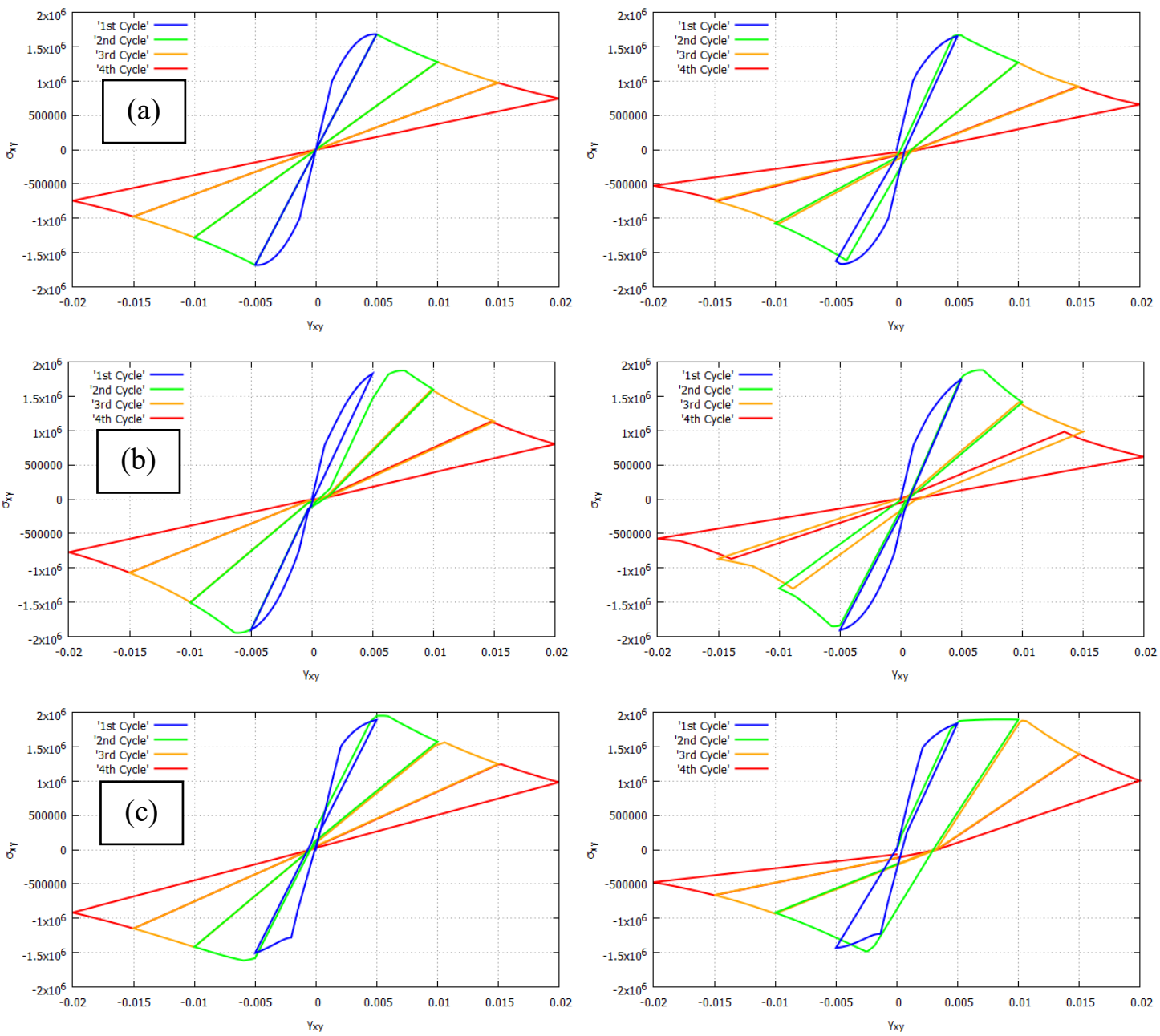

Figure 3. Stress (Pa) vs strain curves of the tests with (a) pure shear, (b) pre-tension and (c) pre-compression with the proposed orthotropic model using $\beta=0$ (left) and $\beta=0.2$ (right)

It is also important to note the comparative performance of the isotropic and orthotropic versions of the model, that is, Figure 1 vs Figure 3. The isotropic model is insensitive to the compressive strength, and damage is only due to the tensile stress that acts in one or the other direction, according to the alternate sign of the applied shear strain. Contrariwise, the orthotropic model responses simultaneously to the principal stresses (tensile and compressive) acting in at the same time. The response under shear of the orthotropic model is a blending of the behavior under pure tension and pure compression, both in terms of strength and dissipation.

The effect that the pre-tension and pre-compression have on the peak value of the stress in both models is as follows. On the one hand, for the isotropic model, for cases (a), in pure shear loading, the peak value of the $\sigma_{x y}$ is $1 \mathrm{MPa}$, which is equal to the tensile strength of the material. However, for cases (b), in pre-tension, the peak value attained is lower than the tensile strength, while for cases (c), under pre-compression, the peak load is noticeably increased. On the other hand, in the orthotropic damage model, the effect of the pre-tension and the pre-compression on the alternate stress peak loads is asymmetrical. 

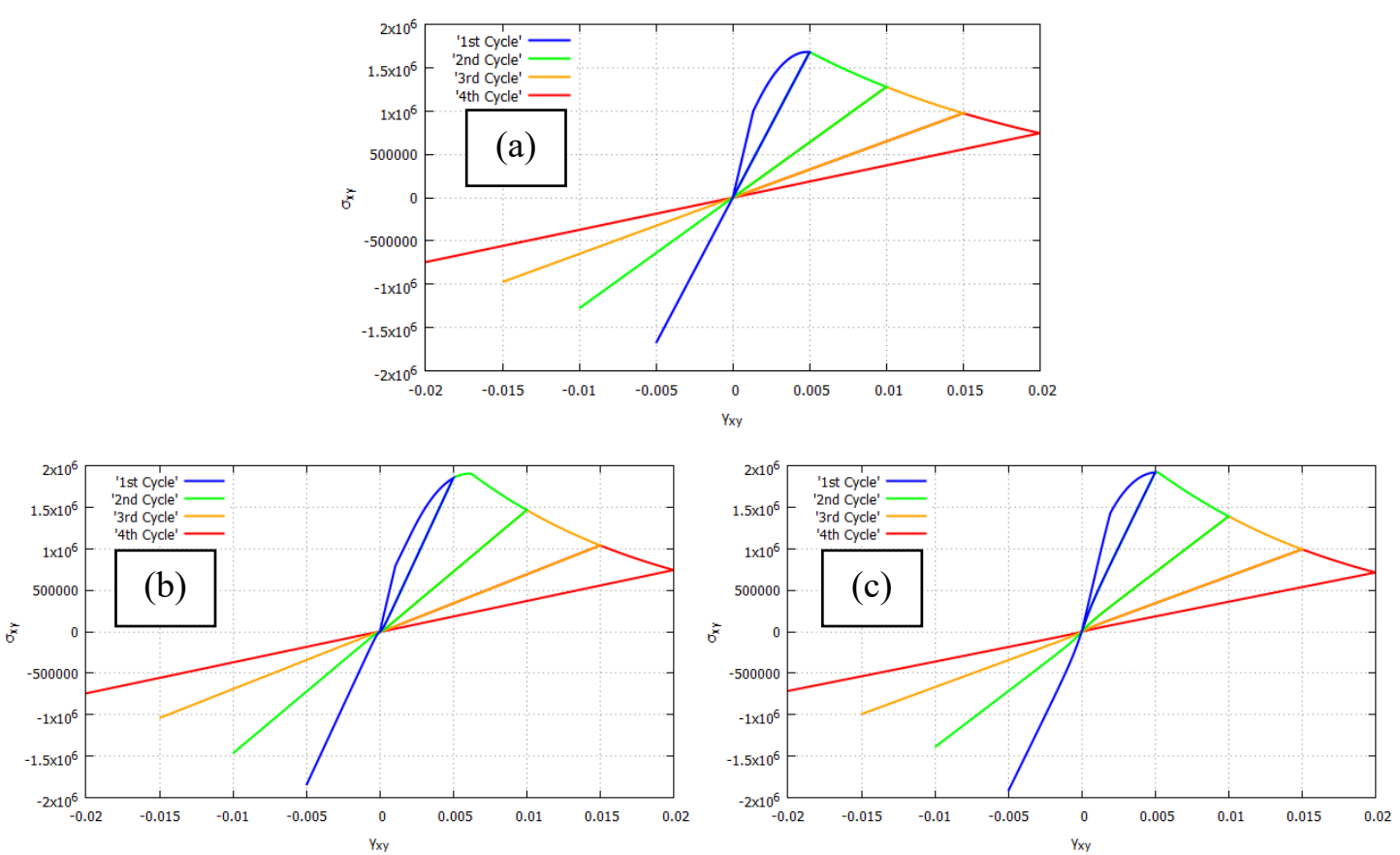

Figure 4. Stress (Pa) vs strain curves of the tests with (a) no pre-compression, (b) low pre-compression and (c) high pre-compression with a classical rotating model
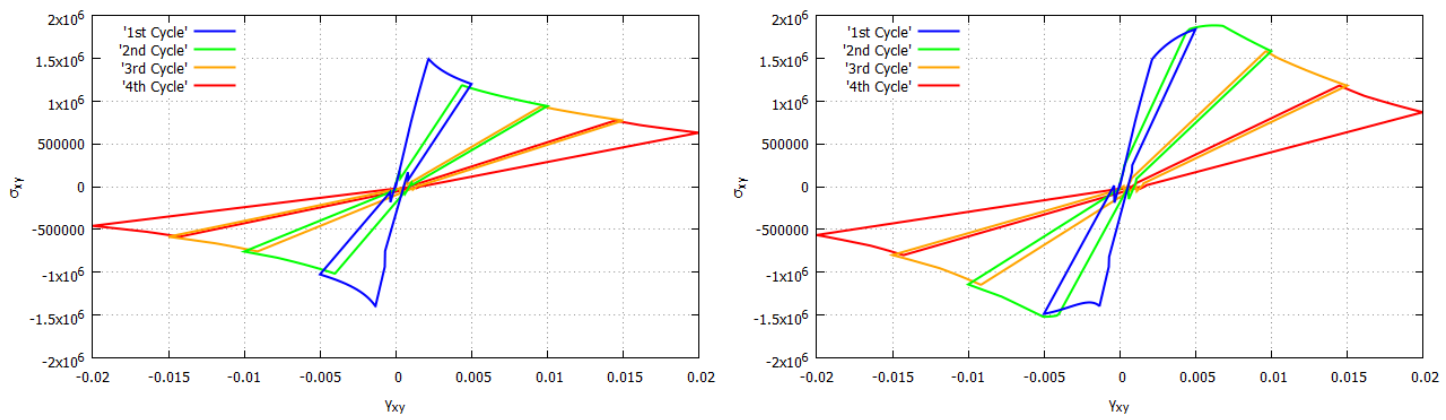

Figure 5. Stress $(\mathrm{Pa})$ vs strain curves of the tests with pre-compression in the isotropic and orthotropic fixed models without irreversible strains of Section 6 guaranteeing stress continuity $(\beta=0.2)$

\section{Mixed FE formulation}

A mixed $\varepsilon / \mathrm{u}$ formulation is used to solve the problem with enhanced strain and stress accuracy and guarantee mesh-objective results. This formulation also helps avoiding the spurious stress oscillations which typically appear in orthotropic models when the standard formulation is used and that usually lead to severe stress locking [12]. In this section, the adopted mixed finite element formulation used to solve the problem is briefly introduced. The mixed FE formulation is described in reference [11]. For additional details on the formulation, references [8, 9, 10, 12, 25] are appropriate.

The model follows the local continuum mechanics framework. In the considered mixed FE formulation, the variational form of the nonlinear solid mechanics problem is computed in terms of the displacement $\boldsymbol{u}$ and the strain $\boldsymbol{\varepsilon}$ fields. Matrix and vector notation based on Voigt's 
convention for symmetric tensors is adopted, as customarily used in FE literature and in most FE codes [19].

The compatibility equation links the strain and displacement fields

$$
\varepsilon=\mathbf{S} \boldsymbol{u}
$$

where $\boldsymbol{S}$ is the differential symmetric gradient operator. Correspondingly, the stress vector $\boldsymbol{\sigma}$ and the body forces vector $\mathbf{f}$ are associated through Cauchy's equilibrium equation, written in matrix form as

$$
\mathbf{S}^{T} \boldsymbol{\sigma}+\mathbf{f}=\mathbf{0}
$$

where $\mathbf{S}^{T}$ is the differential divergence operator, adjoint to the $\mathbf{S}$ in Eq. (41). The constitutive equation relates the stress and strain vectors as

$$
\boldsymbol{\sigma}=\mathbf{D}\left(\varepsilon-\varepsilon^{i}\right)
$$

where $\mathbf{D}$ is the symmetric secant matrix.

A mixed system of equations is obtained pre-multiplying Eq. (41) by the secant matrix $\mathbf{D}$ and substituting Eq. (43) into Eq. (42)

$$
\begin{gathered}
-\mathrm{D} \boldsymbol{\varepsilon}+\mathbf{D S} \boldsymbol{u}=\mathbf{0} \\
\mathbf{S}^{T}\left(\mathbf{D} \boldsymbol{\varepsilon}-\mathbf{D} \boldsymbol{\varepsilon}^{i}\right)+\mathbf{f}=\mathbf{0}
\end{gathered}
$$

The strong form of the mixed $\boldsymbol{\varepsilon} / \boldsymbol{u}$ formulation is defined by the system of Eqs. (44)-(45) and the proper boundary conditions. Note that this differential problem is symmetric if $\mathbf{D}$ is symmetric.

The corresponding weak form is obtained by multiplying Eqs. (44) and (45) by the virtual strain $\delta \boldsymbol{\varepsilon}$ and displacement vector $\delta \boldsymbol{u}$ respectively. The system is then integrated over the spatial domain and the Divergence Theorem is used in the right hand side of the second integral operation. The following variational form results:

$$
\begin{gathered}
-\int_{\Omega} \delta \boldsymbol{\varepsilon}^{T} \mathbf{D} \boldsymbol{\varepsilon} d \Omega+\int_{\Omega} \delta \boldsymbol{\varepsilon}^{T} \mathbf{D S} \boldsymbol{u} d \Omega=0 \quad \forall \delta \boldsymbol{\varepsilon} \\
\int_{\Omega}(\mathbf{S} \delta \boldsymbol{u})^{T}(\mathbf{D} \boldsymbol{\varepsilon}) d \Omega=\int_{\Omega} \delta \boldsymbol{u}^{T} \mathbf{f} \mathrm{d} \Omega+\int_{\Gamma_{t}} \delta \boldsymbol{u}^{T} \overline{\boldsymbol{t}} d \Gamma+\int_{\Omega}(\mathbf{S} \delta \boldsymbol{u})^{T}\left(\mathbf{D} \boldsymbol{\varepsilon}^{i}\right) d \Omega \quad \forall \delta \boldsymbol{u}
\end{gathered}
$$

The last term in the right hand side of Eq. (47) includes the effect of the irreversible strains in the equilibrium equation.

The mixed problem to be solved is to find the unknowns $\boldsymbol{u}$ and $\boldsymbol{\varepsilon}$ that verify the system of Eqs. (46) and (47) and that verify the boundary condition $\boldsymbol{u}=\mathbf{0}$ on $\Gamma_{u}$, for the arbitrary virtual displacements $\delta \boldsymbol{u}$, which vanish on $\Gamma_{u}$, and arbitrary virtual strains $\delta \boldsymbol{\varepsilon}$. Note that this variational problem is symmetric if $\mathbf{D}$ is symmetric.

The FE discrete form of the mixed problem is obtained by discretizing the domain in FE, so that $\Omega=\cup \Omega_{e}$, and substituting the displacement $\boldsymbol{u}$ and the strain $\boldsymbol{\varepsilon}$ with the FE discrete approximations $\widehat{\boldsymbol{u}}$ and $\hat{\boldsymbol{\varepsilon}}$ defined element-wise as

$$
\begin{aligned}
\boldsymbol{u} \cong \widehat{\boldsymbol{u}} & =\boldsymbol{N}_{u} \boldsymbol{U} \\
\boldsymbol{\varepsilon} & \cong \widehat{\boldsymbol{\varepsilon}}=\boldsymbol{N}_{\varepsilon} E
\end{aligned}
$$


where $\boldsymbol{U}$ and $\boldsymbol{E}$ are vectors containing the values of the displacements and the strains at the nodes of the finite element mesh. $\boldsymbol{N}_{u}$ and $\boldsymbol{N}_{\varepsilon}$ are the matrices containing the interpolation functions adopted in the FE approximation.

The Inf-Sup condition is not verified if equal interpolation functions $\boldsymbol{N}_{u}$ and $\boldsymbol{N}_{\varepsilon}$ are used in Eqs. (48)-(49). In that case, the solvability, uniqueness and stability of the solution of the system of equations are ensured by using a stabilization procedure to provide the necessary stability to the mixed discrete formulation. The stabilization procedure consists in the modification of the discrete variational form using the Orthogonal Subscales Method, introduced within the framework of the Variational Multiscale Stabilization methods and adopted herein.

The stabilization procedure is simply to substitute the approximation of the discrete strain in Eq. (49) by the following stabilized discrete field

$$
\boldsymbol{\varepsilon} \cong \widehat{\boldsymbol{\varepsilon}}=\boldsymbol{N}_{\varepsilon} \boldsymbol{E}+\tau_{\varepsilon}\left(\boldsymbol{B}_{u} \boldsymbol{U}-\boldsymbol{N}_{\varepsilon} E\right)=\left(1-\tau_{\varepsilon}\right) \boldsymbol{N}_{\varepsilon} \boldsymbol{E}+\tau_{\varepsilon} \boldsymbol{B}_{u} \boldsymbol{U}
$$

where $\tau_{\varepsilon}$ is a stabilization parameter with value $0 \leq \tau_{\varepsilon} \leq 1$. Note that for $\tau_{\varepsilon}=1$, the strain interpolation of the standard irreducible formulation is recovered:

$$
\varepsilon \cong \widehat{\varepsilon}=B_{u} \boldsymbol{U}
$$

where $\boldsymbol{B}_{u}$ is the discrete strain-displacement matrix defined as $\boldsymbol{B}_{u}=\mathbf{S} \boldsymbol{N}_{u}$

The resulting algebraic system of equations reads:

$$
\left[\begin{array}{cc}
-M_{\tau} & G_{\tau} \\
G_{\tau}^{T} & K_{\tau}
\end{array}\right]\left[\begin{array}{l}
E \\
U
\end{array}\right]=\left[\begin{array}{l}
\mathbf{0} \\
F
\end{array}\right]
$$

where $\left[\begin{array}{ll}\boldsymbol{E} & \boldsymbol{U}\end{array}\right]^{T}$ is the array of nodal values of strains and displacements, and $\boldsymbol{M}_{\boldsymbol{\tau}}=\left(1-\tau_{\varepsilon}\right) \boldsymbol{M}$, $\boldsymbol{G}_{\boldsymbol{\tau}}=\left(1-\tau_{\varepsilon}\right) \boldsymbol{G}$ and $\boldsymbol{K}_{\boldsymbol{\tau}}=\tau_{\varepsilon} \boldsymbol{K} . \boldsymbol{M}$ is a mass like projection matrix, $\boldsymbol{G}$ is the discrete gradient matrix, $\boldsymbol{K}$ is a stiffness like matrix and $\boldsymbol{F}$ is the vector of external nodal forces.

$$
\begin{gathered}
M=\int_{\Omega} N_{\varepsilon}^{T} \mathrm{D} N_{\varepsilon} d \Omega \\
G=\int_{\Omega} N_{\varepsilon}^{T} \mathrm{D} B_{u} d \Omega \\
K=\int_{\Omega} B_{u}^{T} \mathrm{D} B_{u} d \Omega \\
F=\int_{\Omega} N_{u}^{T} f d \Omega+\int_{\Gamma_{t}} N_{u}^{T} \bar{t} d \Gamma+\int_{\Omega} B_{u}^{T} \mathrm{D} \varepsilon^{i} d \Omega
\end{gathered}
$$

In all the simulations shown in Sections 10 and 11, a stabilization parameter $\tau_{\varepsilon}=0.1$ is used. Calculations are performed with an enhanced version of the finite element program COMET [26]. Pre- and post-processing are done with GID [27], developed at CIMNE (International Center for Numerical Methods in Engineering). Convergence of a load step increment is reached when the ratio between the norm of residual forces and the norm of the total external forces is lower than $10^{-3} \%$. 


\section{Concrete beam under cyclic loading}

In this section, the numerical simulation of the test of a concrete beam under cyclic loading is presented. The beam was experimentally tested by [28], which also computed numerical simulations with their proposed constitutive model. Other numerical results are also reported in reference [29], where a non-local formulation is employed.

The objective of this example is to assess the performance of the proposed model with experimental evidence. The geometry of the beam is shown in Figure 6 and the material parameters are given in Table 6. The isotropic Drucker-Prager model is used. The thickness of the beam is $0.05 \mathrm{~m}$. Loads are applied via increments of vertical displacements.

The example is solved under the plane stress hypothesis using two distinct 2D meshes: one of 1998 quadrilateral elements of size $h=5 \mathrm{~mm}$ and a second one of 17,982 quadrilateral elements of size $h=1.66 \mathrm{~mm}$. Both meshes are shown in Figure 7 .

For comparison purposes, the beam in [28] is tested under monotonic and cyclic loading. For this reason, the simulations completed under cyclic loading are also compared with results obtained under a monotonically increasing load.
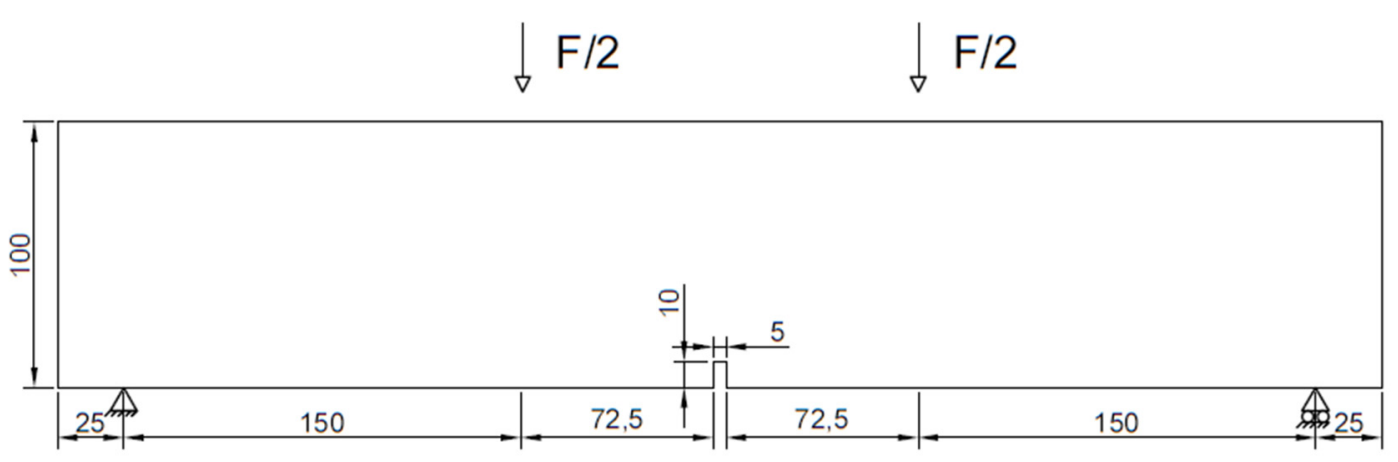

Figure 6. Geometry of the concrete beam under cyclic loading (mm)

\begin{tabular}{|c|c|}
\hline Young's Modulus & $44.0 \cdot 10^{9} \mathrm{~Pa}$ \\
\hline Poisson's Ratio & 0.2 \\
\hline Tensile Strength & $2.35 \cdot 10^{6} \mathrm{~Pa}$ \\
\hline Tensile Fracture Energy & $85 \mathrm{~J} / \mathrm{m}^{2}$ \\
\hline Compressive Strength & $2.35 \cdot 10^{7} \mathrm{~Pa}$ \\
\hline Compressive Fracture Energy & $12000 \mathrm{~J} / \mathrm{m}^{2}$ \\
\hline$\gamma_{0}^{ \pm}$parameter & 1.0 \\
\hline$\gamma_{p}^{ \pm}$parameter & 1.0 \\
\hline$\beta$ parameter for Irreversible Strains & 0.7 \\
\hline
\end{tabular}

Table 6. Material parameters of the concrete beam under cyclic loading 


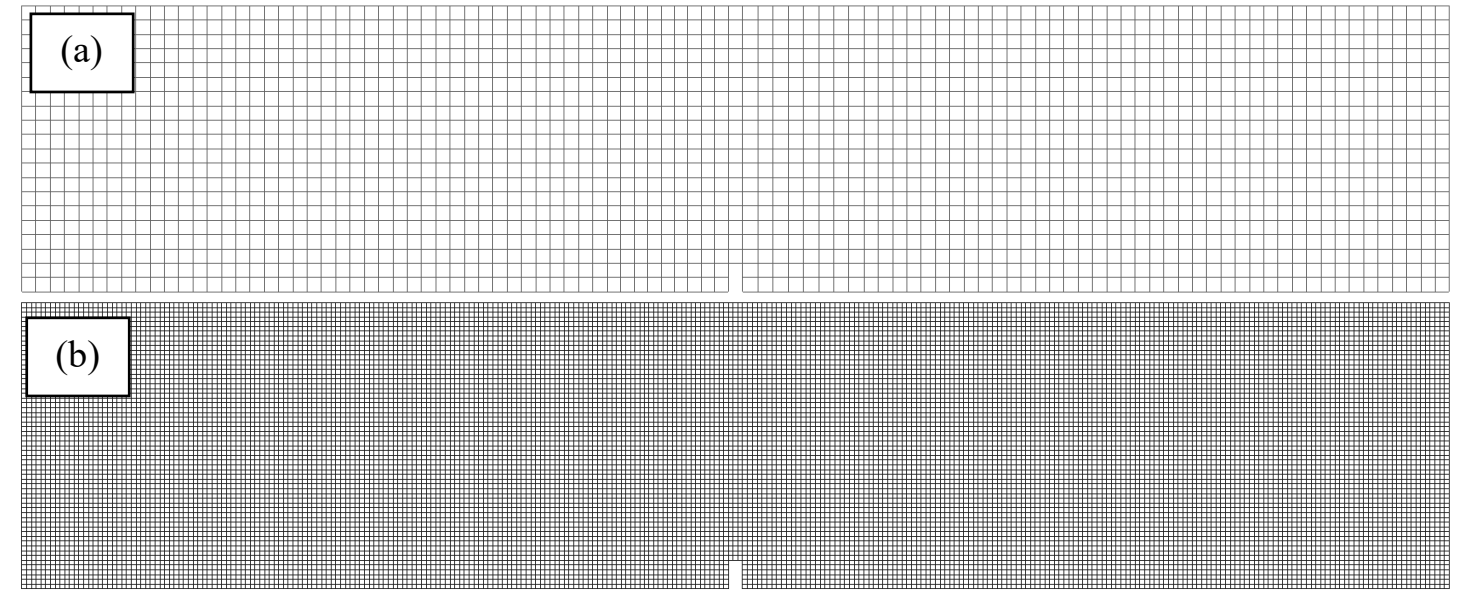

Figure 7. Meshes used for the concrete beam under cyclic loading, (a) $h=5 \mathrm{~mm}$ and (b) $\mathrm{h}=1.66 \mathrm{~mm}$

Figure 8 shows the maximum principal strain map of the computed beams under monotonic loading at the end of the simulation, for an imposed vertical displacement at the midpoint of $\delta=$ $0.7 \mathrm{~mm}$ for both meshes used.

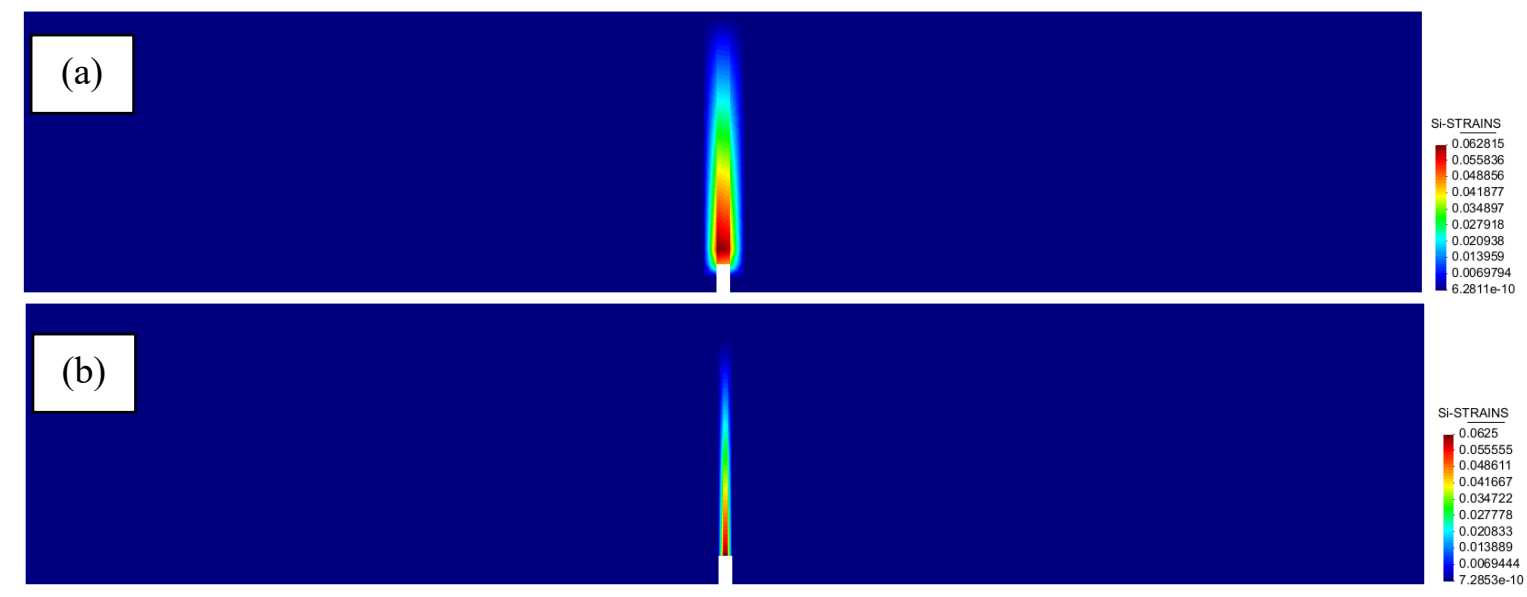

Figure 8. Maximum principal strain field for (a) $h=5 \mathrm{~mm}$ and $(b) h=1.66 \mathrm{~mm}$, for an imposed vertical displacement of $\delta=0.7 \mathrm{~mm}$, in the concrete beam under cyclic loading

Figure 9 shows the force-displacement curves obtained with both meshes are compared. The computed results performed with both meshes are practically overlapping and are very similar to the experimental curves of Reference [28]. For both meshes, the results of the monotonic and the envelope of the cyclic simulations are overlapping. 

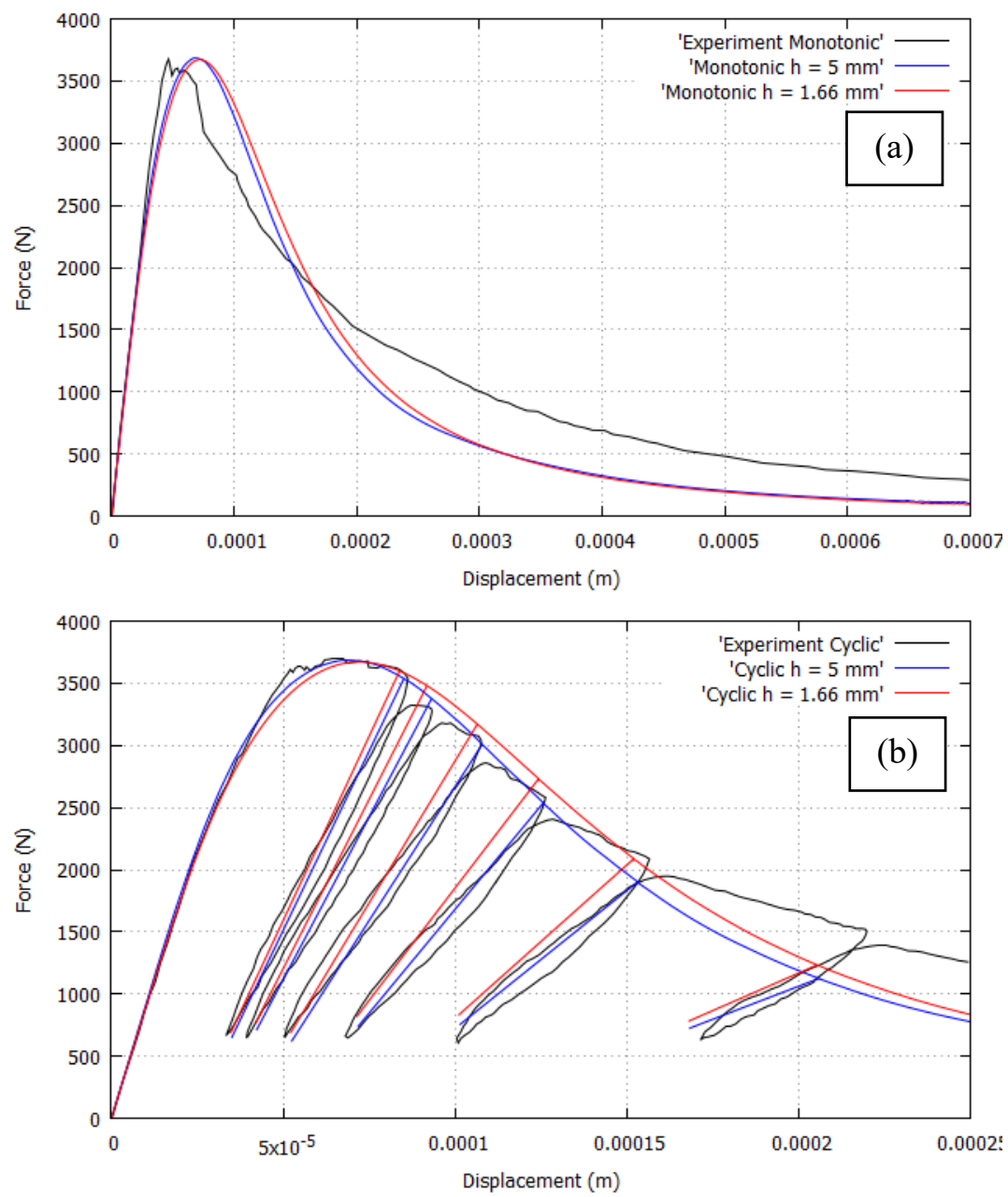

Figure 9. Converge analysis of the (a) monotonic and (b) cyclic simulations of the concrete beam under cyclic loading

\section{Cubic metal specimen under shear cyclic loading}

In this section the numerical simulation of a cubic metal specimen subjected to shear cyclic loading is considered. The objective is to assess the performance of the isotropic and orthotropic proposed models under shear cyclic conditions. For comparison, computations are done with the Rankine and Drucker Prager failure criteria and several different shear loading situations are considered.

The geometry and boundary conditions are shown in Figure 10. A cube metal of size $1 \mathrm{~mm} \times 1$ $m m \times 1 \mathrm{~mm}$ is subjected to monotonic and cyclic shear loading while different levels of precompression are applied A small horizontal slit of $0.1 \mathrm{~mm}$ has been introduced in the center of the cube to fix the occurrence of fracture. The material properties are given in Table 7.

In the tests, the loading is applied via strain increments. Firstly, a pre-compressive strain is applied; secondly several different cycles of shear loading with increasing amplitude are executed. For comparison purposes, the test is performed under different values of initial compressive strain.

The boundary conditions considered are the following. The base of the cube is fixed. The constant pre-compression is applied via a vertical displacement imposed at the top of the cube. The cyclic 
shear load is applied through horizontal displacements also at the top. The vertical displacements of the lateral walls of the cube are prevented.

Many similar versions of this numerical benchmark have been reported for the testing of several crack models, mostly under monotonic conditions, e.g. in [30, 31, 32], where phase-field models are considered. In reference [33] a similar version of the test is used to assess the performance of a fatigue model developed also within the phase-field approach.

The example is solved assuming plane strain behavior and using a 2D structured mesh of $100 \mathrm{x}$ 100 quadrilateral elements of size $h=10^{-5} \mathrm{~m}$.

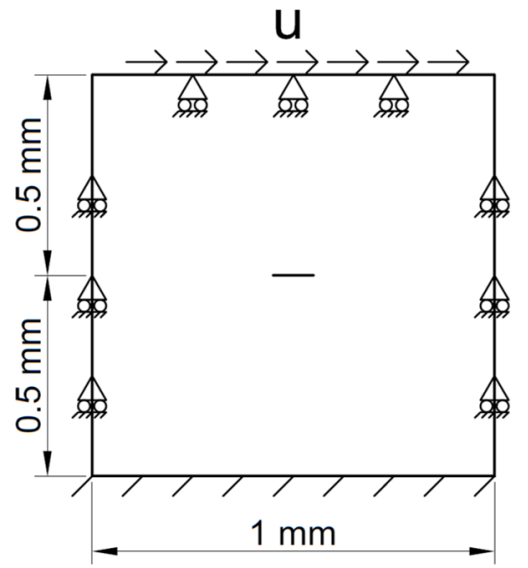

Figure 10. Geometry of the cubic metal specimen under shear cyclic loading

\begin{tabular}{|c|c|}
\hline Young's Modulus & $21.0 \cdot 10^{9} \mathrm{~Pa}$ \\
\hline Poisson's Ratio & 0.3 \\
\hline Tensile Strength & $10^{9} \mathrm{~Pa}$ \\
\hline Tensile Fracture Energy & $30000 \mathrm{~J} / \mathrm{m}^{2}$ \\
\hline Compressive Strength & $30000 \mathrm{~J} / \mathrm{m}^{2}$ \\
\hline Compressive Fracture Energy & 1.0 \\
\hline$\gamma_{0}^{ \pm}$parameter & 1.0 \\
\hline$\gamma_{p}^{ \pm}$parameter & $0.0 / 0.5$ \\
\hline$\beta$ parameter for Irreversible Strains & Various values are considered \\
\hline
\end{tabular}

Table 7. Material parameters of the cubic metal specimen under shear cyclic loading

\subsection{Isotropic Drucker Prager model with varying $f_{c} / f_{t}$ ratio}

The performance of the isotropic Drucker Prager model subjected to pure shear cyclic loading is addressed first. The simulation is done for varying ratios of compressive vs tensile strength. For this, the tensile strength is $f_{t}=10^{9} \mathrm{~Pa}$, while the ratio $f_{c} / f_{t}=1,5,8$ and 100 .

Figure 11 shows the computed crack trajectories for different ratios of compressive vs tensile strength under monotonic pure shear loading. It can be seen how for the case $f_{c} / f_{t}=1$ the crack trajectory is a horizontal straight line, as is typical in metals for this type of loading and purely deviatoric behavior. For increasing values of $f_{c} / f_{t}$ the crack turns and tends to a trajectory at an angle of $45^{\circ}$ with respect the horizontal axis for the case $f_{c} / f_{t}=1$, as would happen for cementitious materials. 


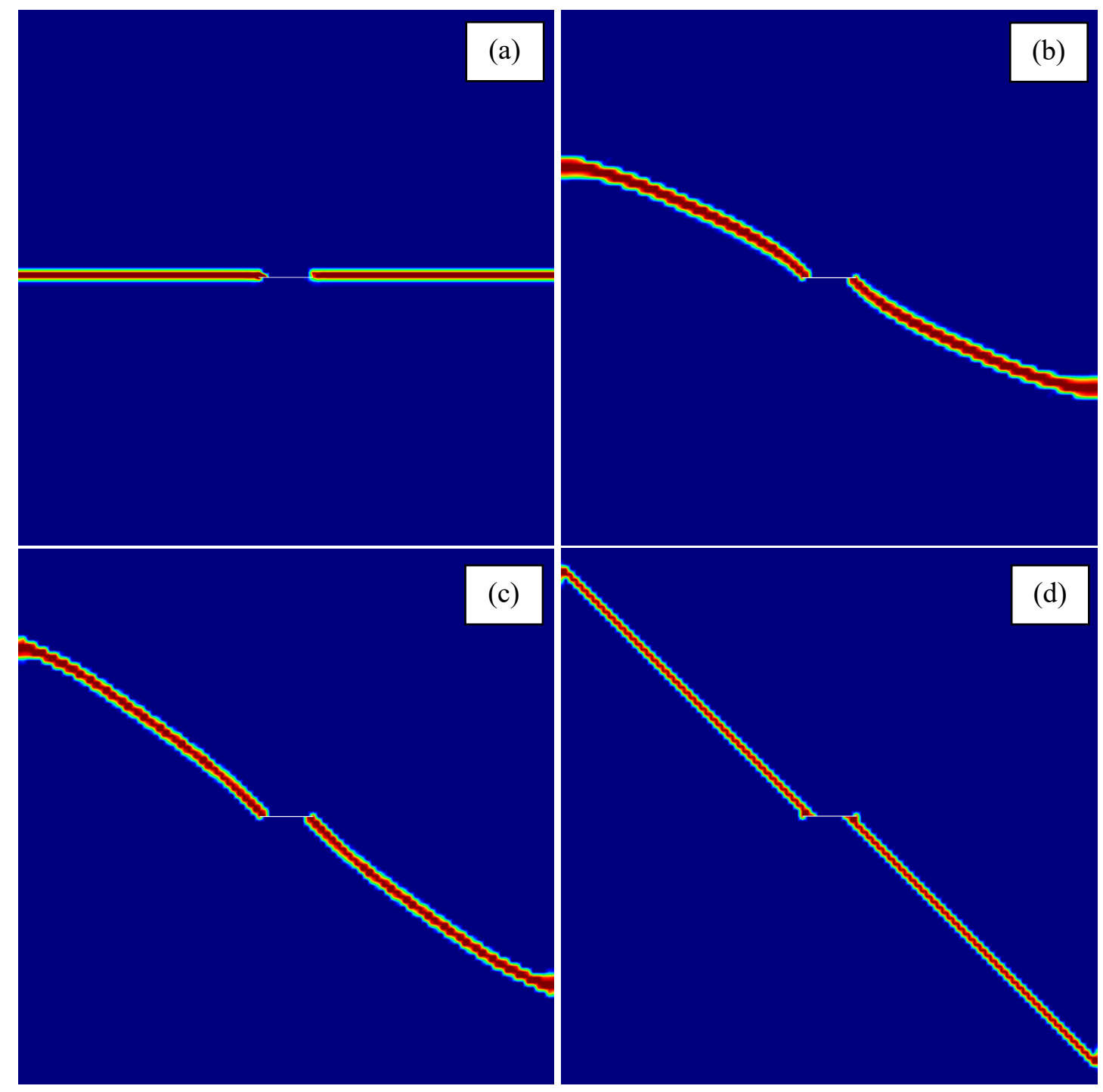

Figure 11. Isotropic Drucker Prager model damage contours for the cubic metal specimen under monotonic loading with (a) $f_{c} / f_{t}=1$, (b) $f_{c} / f_{t}=5$, (c) $f_{c} / f_{t}=8$ and (d) $f_{c} / f_{t}=100$ 


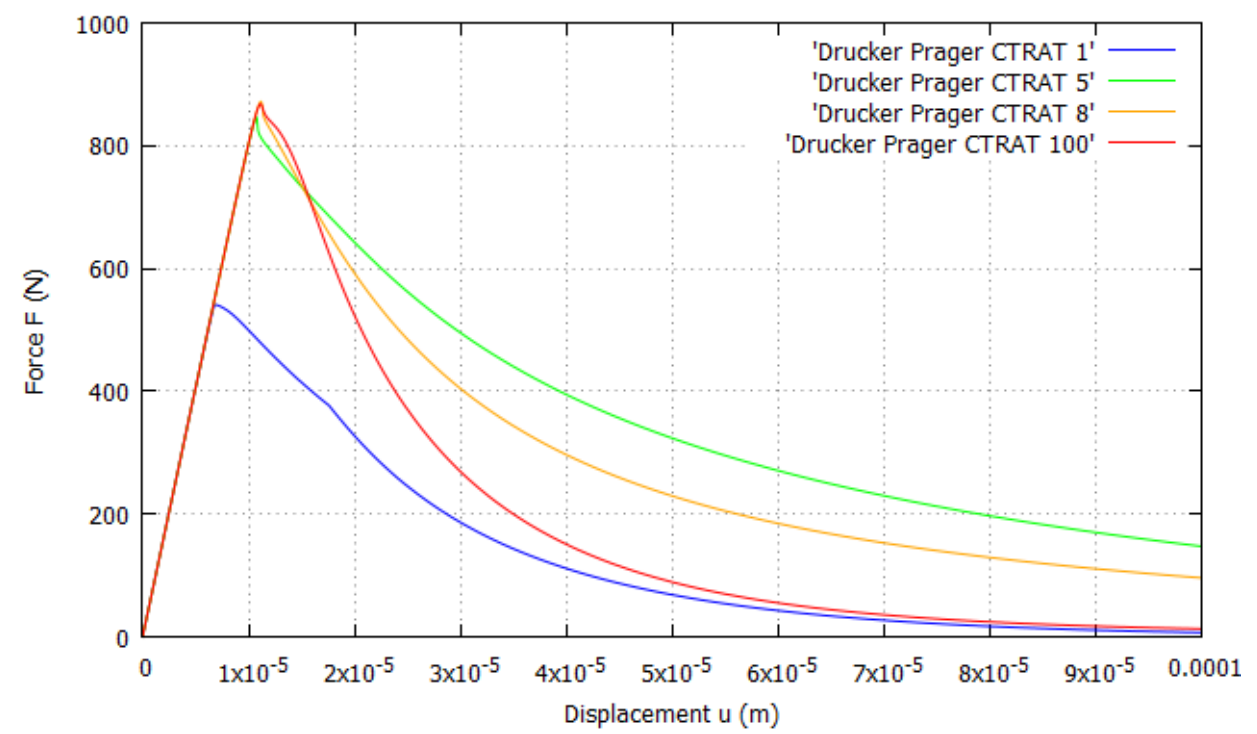

Figure 12. Isotropic Drucker-Prager model force-displacement curves for the different ratios of compressive vs tensile strength

It is to be noted that for the reciprocal ratios $f_{c} / f_{t}=1 / 5,1 / 8$ and $1 / 100$, with the compressive strength smaller than the tensile one, the exact skew symmetric crack trajectories with respect the ones shown in Figure 11 develop.

In Figure 12 the force-displacement curves of the different situations are presented. It can be seen how the peak value of the force increases as the $f_{c} / f_{t}$ ratio is increased, with the case $f_{c} / f_{t}=1$ reaching a much lower peak value than the other cases. It can also be appreciated how the behavior of the material becomes more brittle as the $f_{c} / f_{t}$ ratio increases.

Figure 13 shows the crack trajectories for different ratios of compressive vs tensile strength under monotonic (left) and cyclic (right) pure shear loading. It can be seen how the cracks under cyclic loading have central symmetry with respect the center of the sample and that cracks are nearly overlapping with the respective monotonic result. The observed differences in the trajectories between monotonic and cyclic results are of 1 element at the most, the resolution of the mesh.

In Figure 14 it can be seen how the force-displacement curves of the monotonic and cyclic simulations are overlapping in all the situations. It can also be seen how the stiffness is properly and completely recovered upon load reversal. 


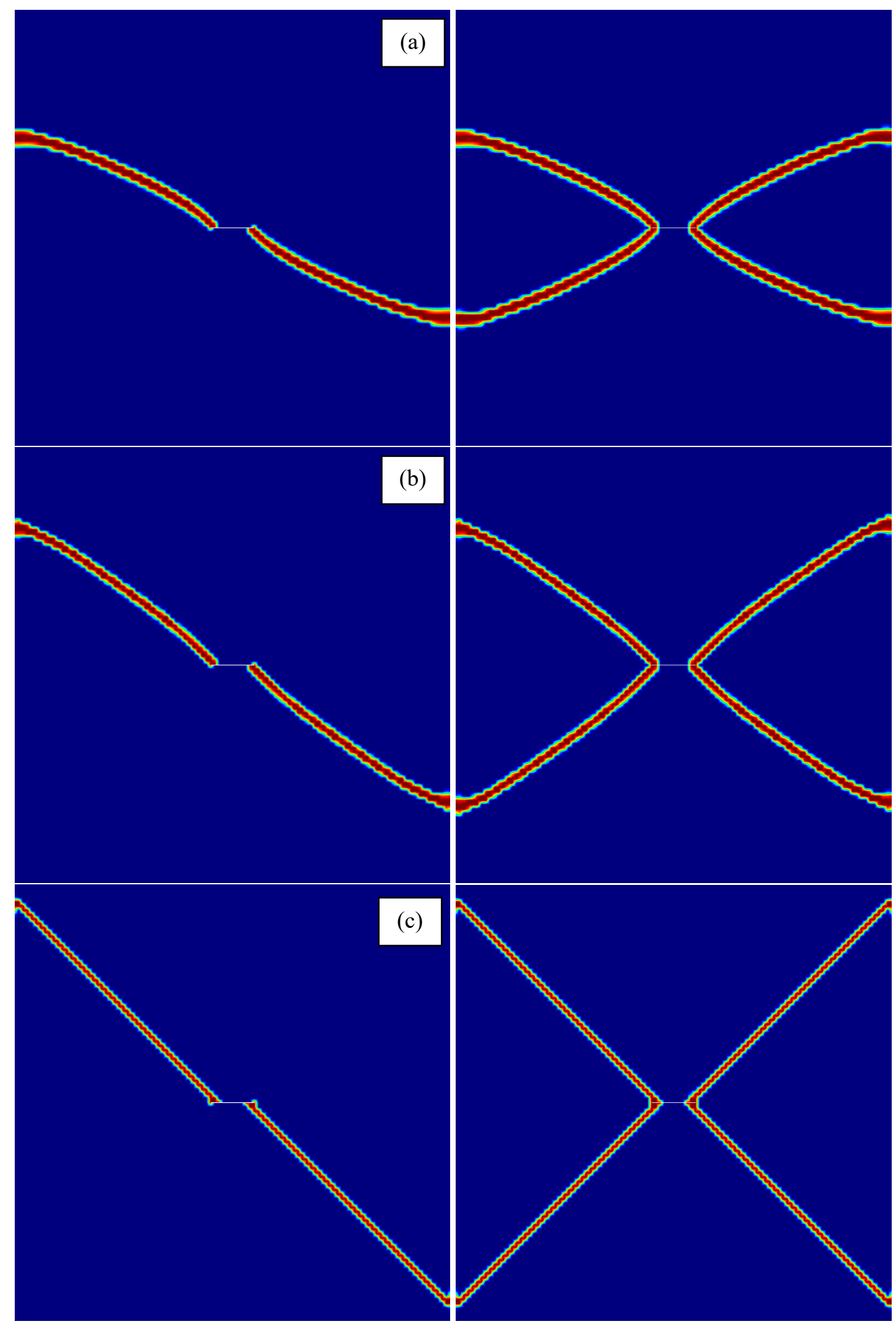

Figure 13. Isotropic Drucker-Prager model damage contours for the cubic metal specimen under monotonic (left) and cyclic (right) loading with (a) $f_{c} / f_{t}=5$, (b) $f_{c} / f_{t}=8$ and (c) $f_{c} / f_{t}=100$ 

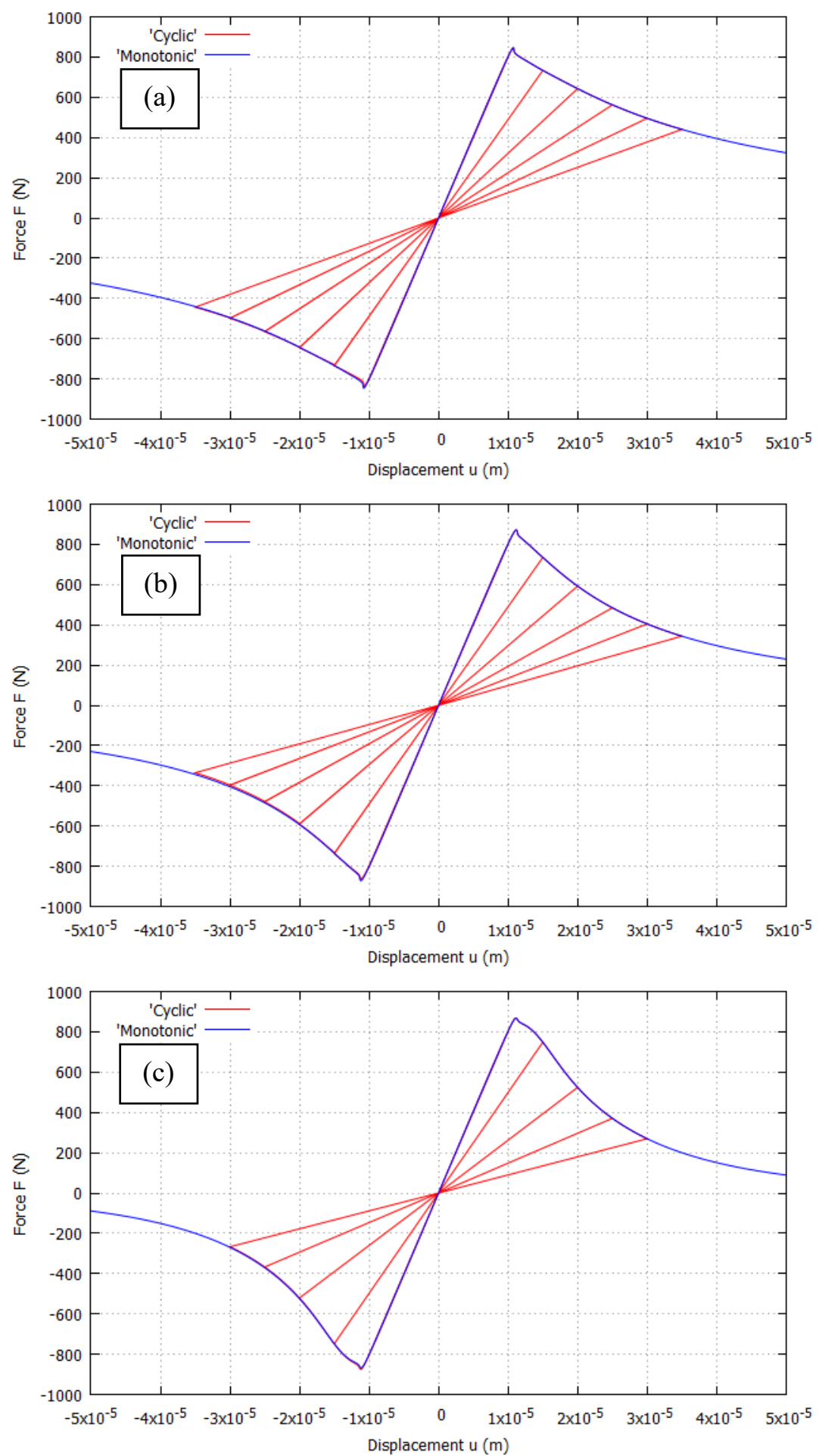

Figure 14. Isotropic Drucker Prager model force-displacement curves for the cubic metal specimen under monotonic and cyclic loading with (a) $f_{c} / f_{t}=5$, (b) $f_{c} / f_{t}=8$ and (c) $f_{c} / f_{t}=100$ 


\subsection{Isotropic vs Orthotropic Rankine model}

The comparison of the isotropic and orthotropic Rankine models subjected to pure shear is now assessed. For the sake of clarity, $f_{c} / f_{t}=\infty$, and only tensile damage is considered. Figure 15 shows the crack trajectories for isotropic and orthotropic Rankine under monotonic (left) and cyclic (right) pure shear loading, demonstrating that crack trajectories are neatly different for isotropic and orthotropic damage. The force-displacement curves are depicted in Figure 16. It is remarkable that isotropic models may soften completely under shear loading, while orthotropic models fully retain their stiffness in the undamaged directions. Results are again overlapping in monotonic and cyclic loadings situations. The stiffness is properly recovered when cracks close and re-open.

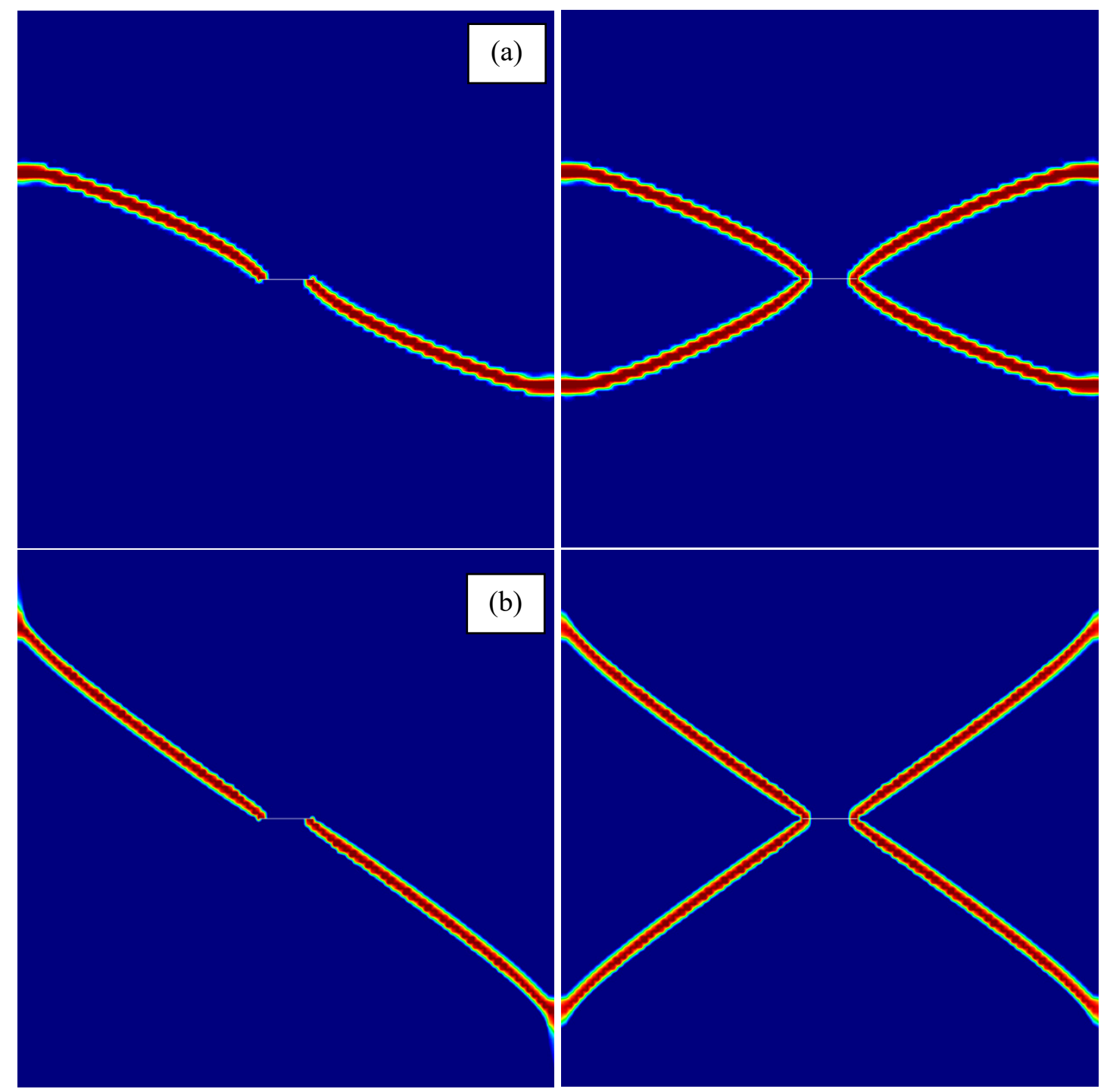

Figure 15. Rankine model damage contours of the (a) isotropic and (b) orthotropic version for the cubic metal specimen under monotonic (left) and cyclic (right) loading 

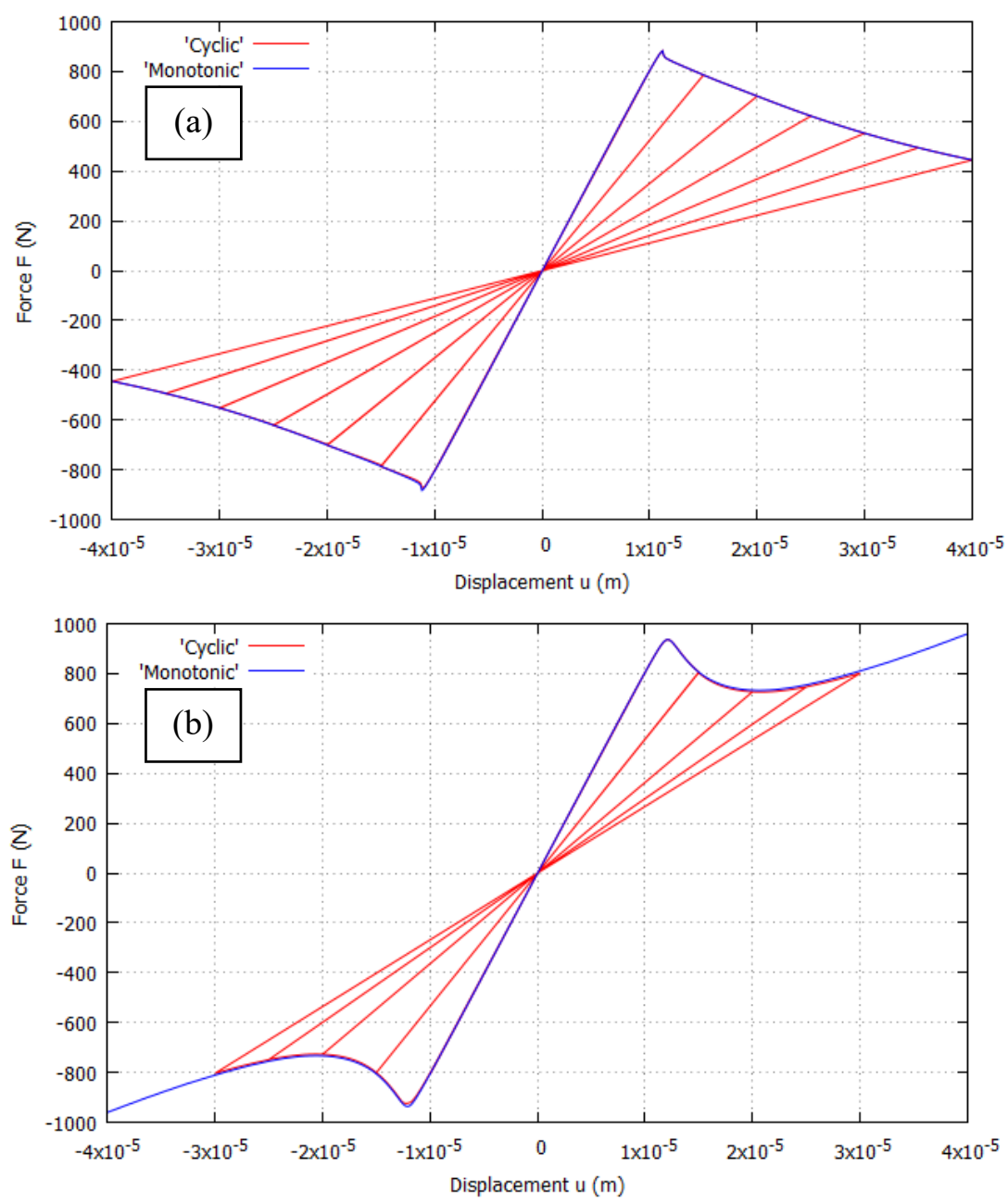

Figure 16. (a) Isotropic and (b) orthotropic Rankine model force-displacement curves for the cubic metal specimen under monotonic and cyclic loading

\subsection{Effect of the pre-compression in the cyclic behavior}

In this section, the performance of the model is assessed in situations in which the cubic metal specimen is first subjected to a level of pre-compression before the cycles of shear load of increasing amplitude are applied. The results obtained under three different values of precompressive strain, noted as $\varepsilon_{c}$, are compared:

- Pure shear cyclic loading test without compression, $\varepsilon_{c}=0$

- Low compression, $\varepsilon_{c}=2.0 \cdot 10^{-3}$

- High compression, $\varepsilon_{c}=4.0 \cdot 10^{-3}$

The isotropic Rankine model is used, with $f_{c} / f_{t}=\infty$. Therefore, only tensile damage is considered. 


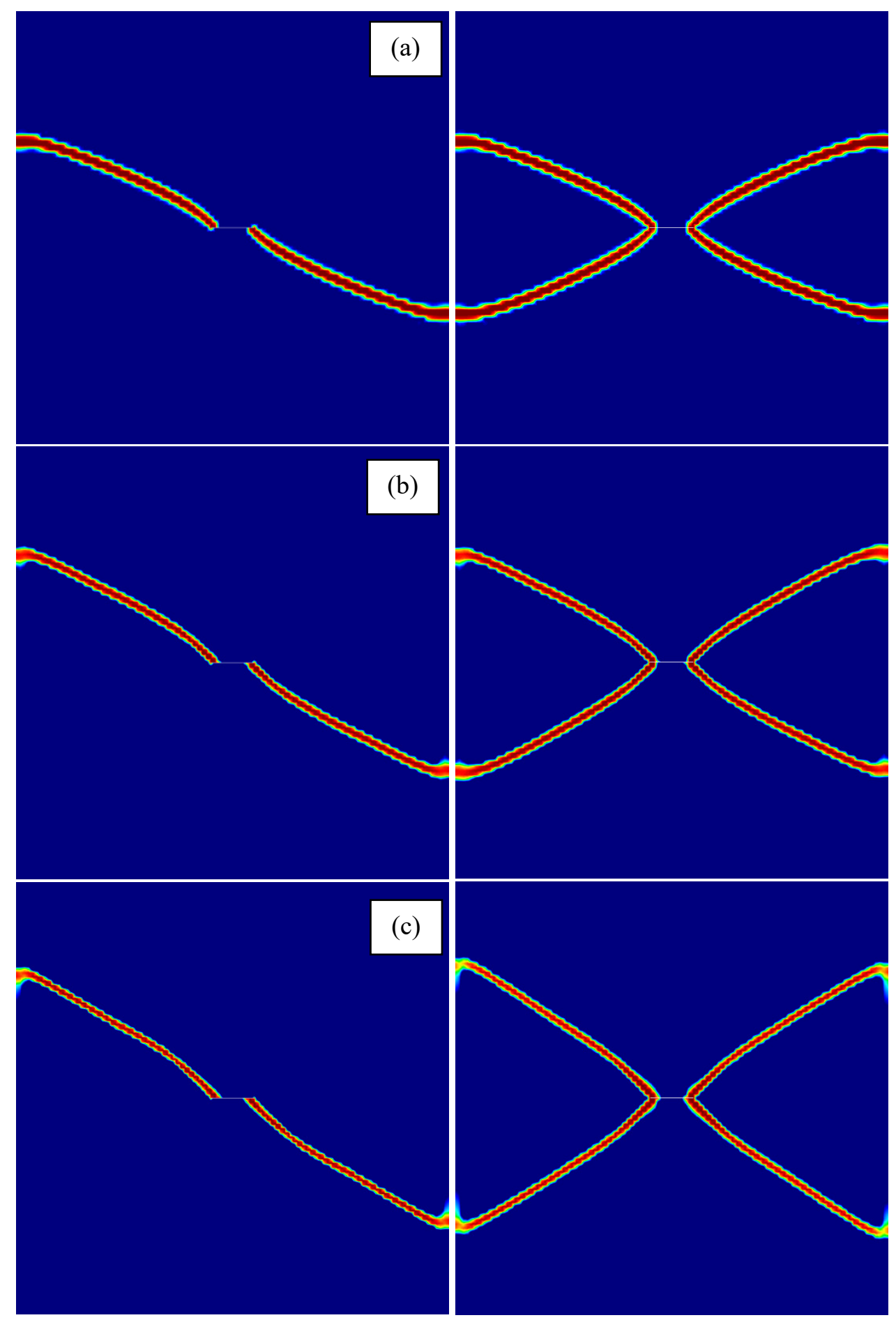

Figure 17. Isotropic Rankine model damage contours with (a) no compression, (b) low compression and (c) high compression for the cubic metal specimen under monotonic (left) and cyclic (right) loading 


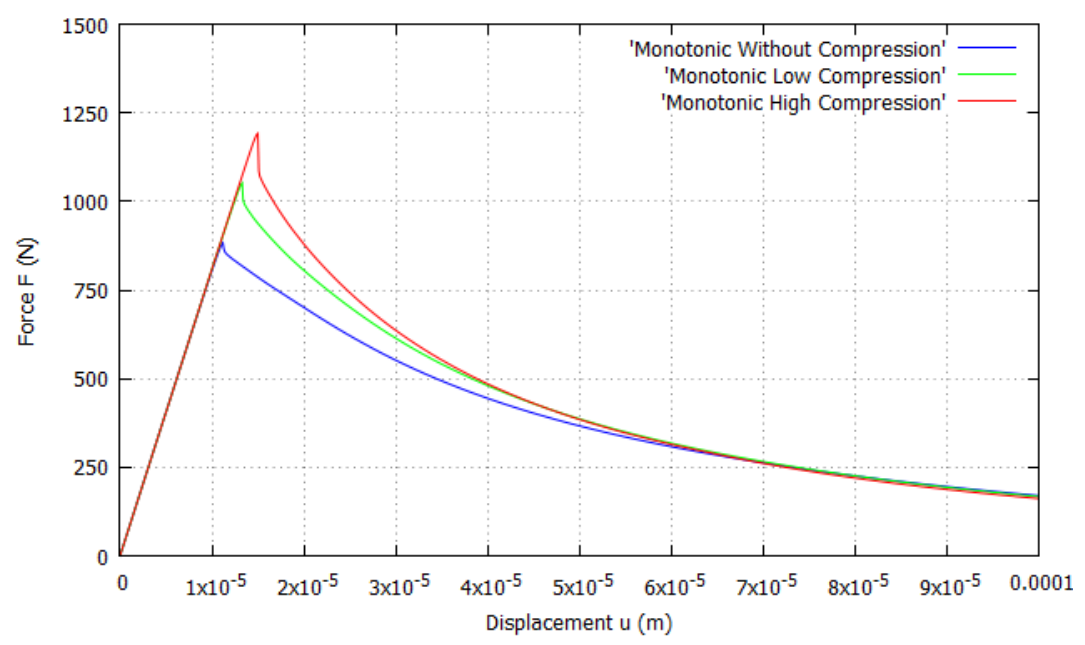

Figure 18. Isotropic Rankine model force-displacement curves with different pre-compression levels under monotonic loading in the cubic metal specimen
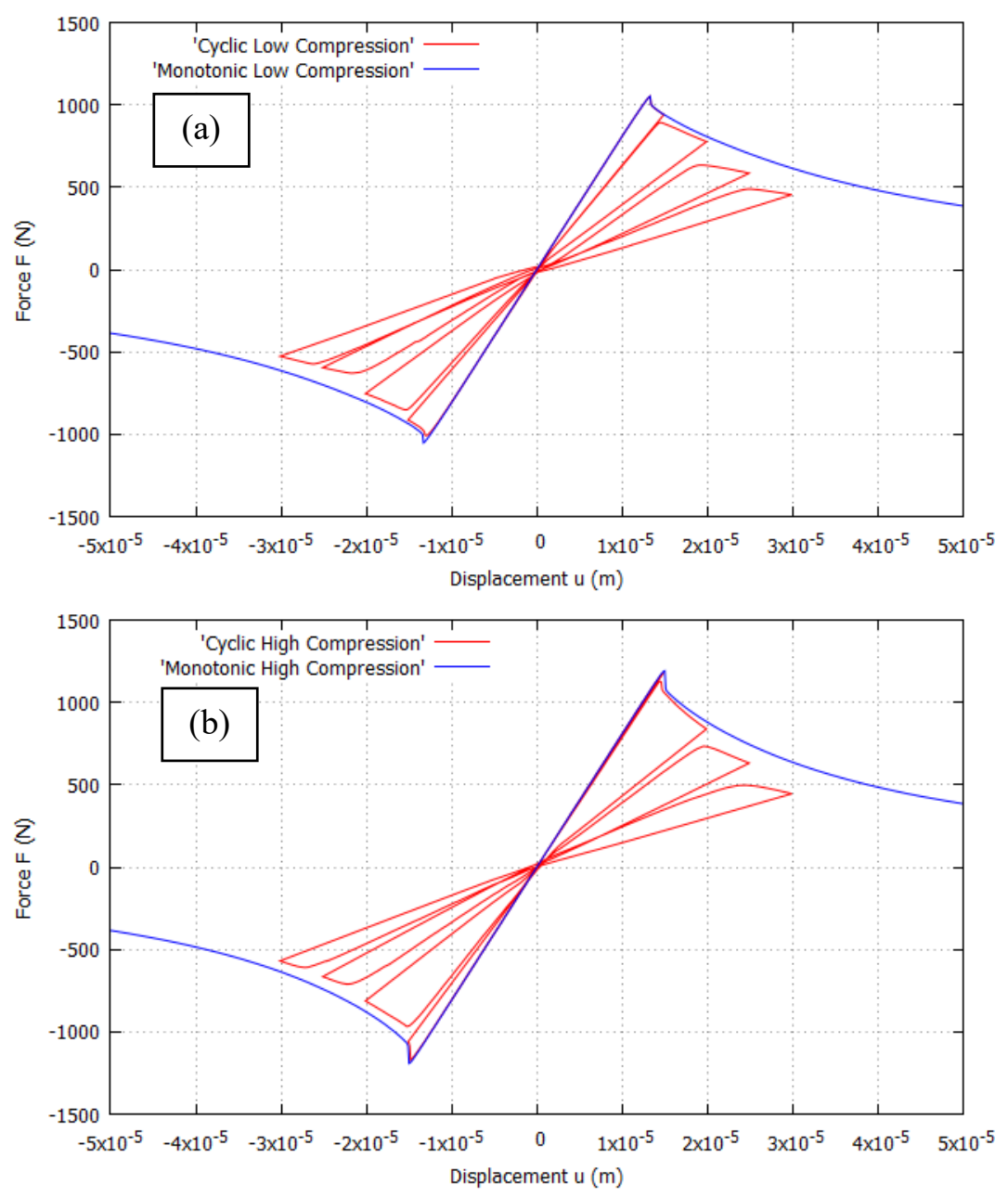

Figure 19. Isotropic Rankine model force-displacement curves for the cubic metal specimen under monotonic and cyclic loading in the (a) low compression and (b) high compression case 
Figure 17 shows the crack trajectories obtained with different levels of pre-compression under monotonic (left) and cyclic (right) loading. It can be seen how the crack trajectories vary in function of the pre-compression applied to the cubic specimen. The in-between angle formed by the cracks increases with the pre-compression.

Figure 18 depicts the force-displacement curves under monotonic loading of the three cases considered. It can be seen how the peak load of the specimen increases when the pre-compression is increased.

In Figure 19, the monotonic and cyclic force-displacement curves are compared in the low and high pre-compression cases. It can be observed that stiffness is duly recovered when cracks close and reopen.

These results are to be compared with the curves of Figure 16a where no pre-compression is applied. It can be seen that the monotonic and cyclic curves no longer overlap when precompression is applied. This is caused by the development and accumulation of "sealing" strains when the cracks close or reopen. This causes a loss of symmetry in the force-displacement curves. The slight loss of symmetry caused by the "sealing" strains can also be observed in Figure 17 in the two cracks that develop in the body.

\subsection{Effect of the irreversible strains}

In this section, the effect of irreversible strains is included in the simulations. To this end, compressive damage is also considered in the isotropic Rankine model and a ratio $f_{c} / f_{t}=3$ is used. The parameter $\beta=0.5$ is used for irreversible strains. The same three loading cases with different pre-compression levels from the previous section are examined.

Figure 20 shows the crack trajectories obtained with different levels of pre-compression under monotonic (left) and cyclic (right) loading with the development of irreversible strains. The crack trajectories deviate in function of the level of pre-compression initially applied to the specimen. The results differ very slightly from those in Figure 17.

In Figure 21 the monotonic and cyclic force-displacement curves are depicted. It can be seen how in the cyclic results the development of irreversible strains under compression causes a noticeable loss of symmetry and the corresponding deviation with respect the monotonic computations. 


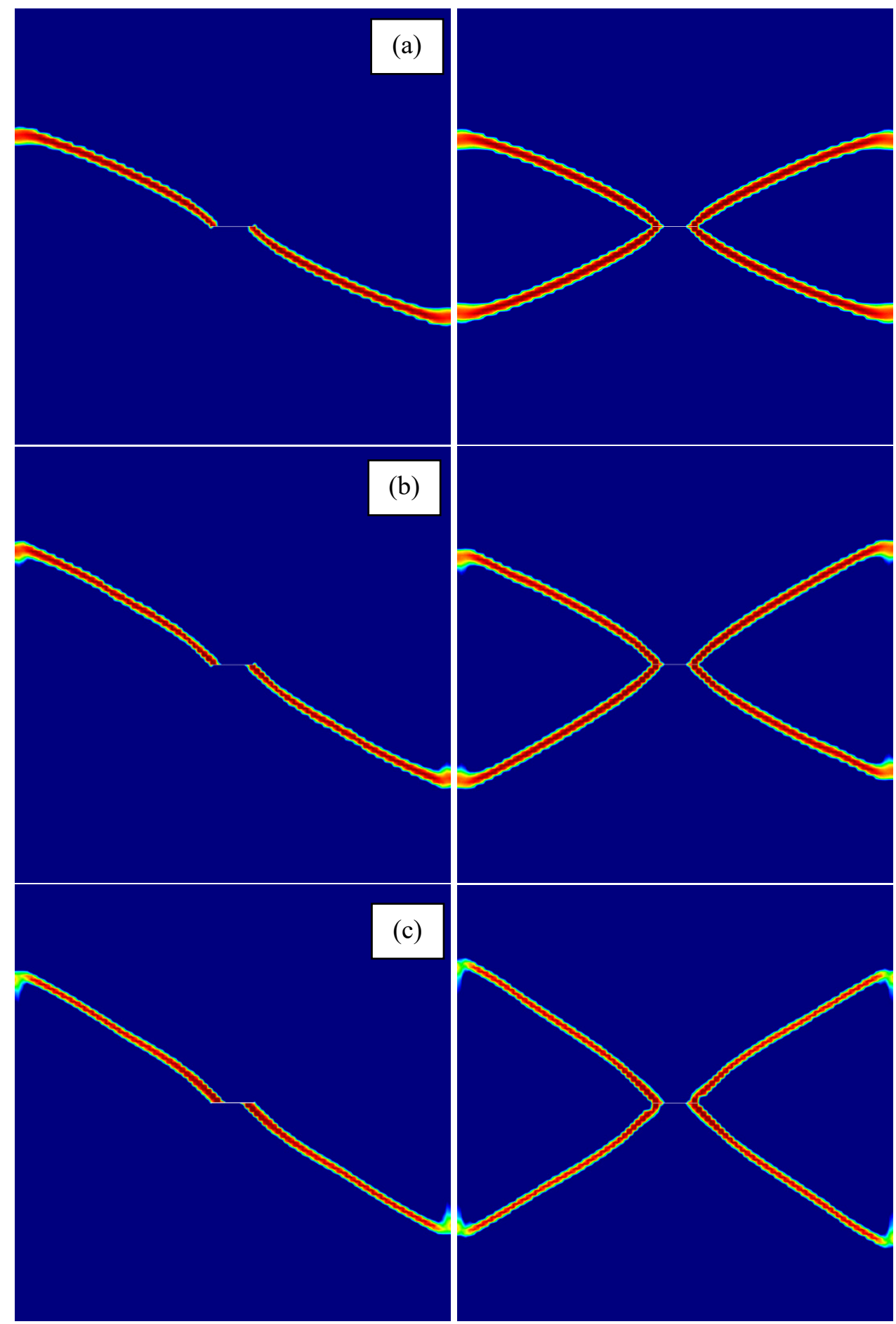

Figure 20. Isotropic Rankine model damage contours of the with irreversible strains $(\beta=0.5)$ with (a) no compression, (b) low compression and (c) high compression for the cubic metal specimen under monotonic (left) and cyclic (right) loading 

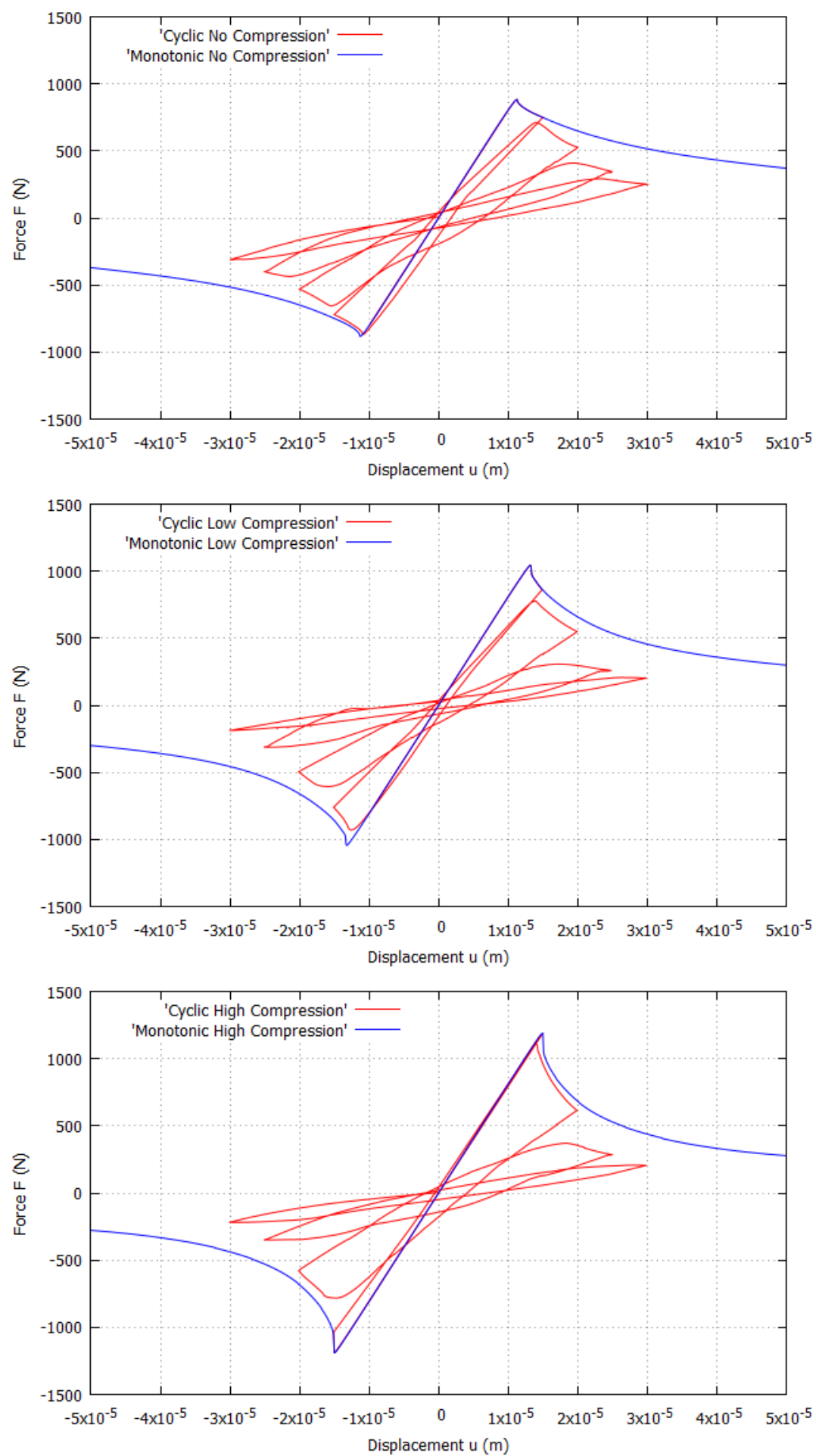

Figure 21. Isotropic Rankine model with irreversible strains $(\beta=0.5)$ force-displacement curves for the cubic metal specimen under monotonic and cyclic loading in the (a) no compression, (b) low compression and (c) high compression case 


\section{Conclusions}

In this work, a novel multi-crack model with full closing, reopening and sliding capabilities is proposed. The crack model is assembled from different features, some typical from classical orthogonal crack models, some others from up-to-date damage-based models, and some novel components. These are purposefully added to model micro-crack closure-reopening effects with significant sliding (MCRS). Two versions of the model, isotropic and orthotropic, are proposed, both provided with MCRS capabilities.

The model is used in conjunction with a mixed $\boldsymbol{\varepsilon} / \mathbf{u}$ finite element formulation which provides enhanced accuracy and mesh-independent results in cracking problems.

Numerical benchmarks are used to demonstrate the performance of the model in Mode I, Mode II and Mixed Mode I and II cyclic loading situations.

It is observed that:

- The proposed damage constitutive models are fit for the numerical simulation of cracks in quasi-brittle materials with MCRS effects under cyclic loading situations.

- The phenomena of tensile and compressive damage, stiffness recovery and irreversible strains are properly reproduced.

- The model, used together with the mixed FE formulation is able to reproduce the behavior observed in experimental tests as well as to emulate complex crack patterns with MCRS effects.

- Results obtained are free from the spurious mesh bias and stress-locking typical of standard FEs.

- In mode I (traction) and in pure shear situations, monotonic and cyclic results obtained with the models are perfectly overlapping in terms of cracks trajectories and forcedisplacement curves.

- With pre-loading, monotonic and cyclic results are not overlapping, as irreversible strains develop and accumulate. This effect is very evident in force-displacement curves.

- Distinct results are obtained for different damage models. Hence, different damage criteria produce different crack trajectories and force-displacement curves.

- Likewise, isotropic and orthotropic versions of the same damage criterion also show very different results.

From these, it is concluded that the proposed isotropic and orthotropic constitutive damage models with memory, used in conjunction with the mixed finite element formulation, are capable of computing mode I, mode II and mixed mode I and II cracking problems taking into consideration MCRS effects and correctly computing stiffness recovery under cyclic loadings with shear sliding.

\section{Acknowledgements}

Financial support from the Spanish Ministry of Economy and Business via the ADaMANT (Computational Framework for Additive Manufacturing of Titanium Alloy) project (Proyectos de I + D (Excelencia) DPI2017-85998-P) is gratefully acknowledged. The support provided by the Spanish Ministry of Education to Mr. Gabriel Barbat via the FPU program is also acknowledged. 


\section{References}

[1] D. Ngo and A. Scordelis, "Finite element analysis of reinforced concrete beams," American Concrete Institute Journal, vol. 64, no. 14, pp. 152-163, 1967.

[2] Y. Rashid, "Ultimate strength analysis of prestressed concrete pressure vessels," Nuclear Engineering and Design, vol. 7, no. 4, pp. 334-344, 1968.

[3] S. Saloustros, M. Cervera and L. Pelà, "Challenges, tools and applications of tracking algorithms in the numerical modelling of cracks in concrete and masonry structures," Archives of Computational Methods in Engineering, https://doi.org/10.1007/s11831-0189274-3, 2018.

[4] R. Litton, "A contribution to the analysis of concrete structures under cyclic loading," Ph.D. Thesis, University of California, Berkeley, 1975.

[5] R. de Borst and P. Nauta, "Non-orthogonal cracks in a smeared finite element model," Engineering Computations, vol. 2, pp. 35-46, 1985.

[6] Z. Bazant and P. Gambarova, "Crack shear in concrete: Crack band microplane model," Journal of Structural Engineering, vol. 110, no. 9, pp. 2015-2036, 1984.

[7] I. Carol, P. Prat and Z. Bazant, "New explicit microplane model for concrete: theoretical aspects and numerical implementation," International Journal of Solids and Structures, vol. 29, no. 9, pp. 1173-1191, 1992.

[8] M. Cervera, M. Chiumenti and R. Codina, "Mixed stabilized finite element methods in nonlinear solid mechanics. Part I: Formulation," Computer Methods in Applied Mechanics and Engineering, vol. 199, no. 37-40, pp. 2559-2570, 2010.

[9] M. Cervera, M. Chiumenti and R. Codina, "Mixed stabilized finite element methods in nonlinear solid mechanics. Part II: Strain localization," Computer Methods in Applied Mechanics and Engineering, vol. 199, no. 37-40, pp. 2571-2589, 2010.

[10] M. Cervera, M. Chiumenti, L. Benedetti and R. Codina, "Mixed stabilized finite element methods in nonlinear solid mechanics. Part III: Compressible and incompressible plasticity," Computer Methods in Applied Mechanics and Engineering, vol. 285, pp. 752$775,2015$.

[11] M. Cervera, G. Barbat and M. Chiumenti, "Finite element modelling of quasi-brittle cracks in 2D and 3D with enhanced strain accuracy," Computational Mechanics, vol. 60, no. 5, pp. 767-796, 2017.

[12] G. Barbat, M. Cervera and M. Chiumenti, "Appraisement of planar, bending and twisting cracks in 3D with isotropic and orthotropic damage models," International Journal of Fracture, vol. 210, no. 1-2, pp. 45-79, 2018.

[13] G. Vlachakis, M. Cervera, G. Barbat and S. Saloustros, "Out-of-plane seismic response and faillure mechanism of masonry structures using finite elements with enhanced strain accuracy," Engineering Faillure Analysis, vol. 97, pp. 534-555, 2019. 
[14] R. Faria, J. Oliver and M. Cervera, "A strain-based plastic viscous-damage model for massive concrete structures," International Journal of Solids and Structures, vol. 35, no. 14, pp. 1533-1558, 1998.

[15] C. Comi and U. Perego, "Fracture energy based bi-dissipative model for concrete," International Journal of solids and structures, vol. 38, no. 36-37, pp. 6427-6454, 2001.

[16] R. Faria, J. Oliver and M. Cervera, "Modeling material failure in concrete structures under cyclic actions," Journal of Structural Engineering, vol. 130, no. 12, pp. 1997-2005, 2004.

[17] M. Cervera and C. Tesei, "An energy-equivalent $\mathrm{d}+/ \mathrm{d}$ - damage model with enhanced microcrack closure-reopening capabilities for cohesive-frictional materials," Materials, vol. 10, no. 4, p. 433, 2017.

[18] M. Cervera, C. Tesei and G. Ventura, "Cracking of quasi-brittle structures under monotonic and cyclic loadings: a d+/d- damage model with stiffness recovery in shear," International Journal of Solids and Structures, vol. 135, pp. 148-171, 2017.

[19] O. Zienkiewicz, R. Taylor and Z. Zhu, "The finite element method, Vol. 1," 7th edition, Amsterdam, Elsevier Butterworth-Heinemann, 1989.

[20] J. Oliver, M. Cervera, S. Oller and J. Lubliner, "Isotropic damage models and smeared crack analysis of concrete," II international conference on computer aided analysis and design of concrete, 1990.

[21] G. Voyiadjis, Z. Taqieddin and P. Kattan, "Anisotropic damage-plasticity model for concrete," International Journal Plasticity, vol. 24, pp. 1946-1965, 2008.

[22] J. Cordebois and F. Sidoroff, "Endommagement anisotrope en elasticité et plasticité," Journal de Mecanique Theorique et Appliquee, vol. Special Volume, pp. 45-60, 1982.

[23] I. Carol, E. Rizzi and K. Willam, "On the formulation of anisotropic elastic degradation. I: Theory based on pseudo-logarithmic damage tensor rate; II: Generalized pseudo-Rankine model for tensile damage," International Journal of Solids and Structures, vol. 38, no. 4, pp. 491-546, 2001.

[24] S. Saloustros, M. Cervera and L. Pela, "Tracking multi-directional intersecting cracks in numerical modelling of masonry shear walls under cyclic loading," Meccanica, Special Issue on "New Trends in Mechanics of Masonry", http://dx.doi.org/10.1007/s11012-0170712-3, 2017.

[25] L. Benedetti, M. Cervera and M. Chiumenti, "3D numerical modelling of twisting cracks under bending and torsion skew notched beams," Engineering Fracture Mechanics, vol. 176, pp. 235-256, 2017.

[26] M. Cervera, C. Agelet de Saracibar and M. Chiumenti, "COMET: Coupled Mechanical and Thermal Analysis. Data Input Manuel, Version 5.0, Technical report IT-308. Available from http://www.cimne.upc.edu," 2002.

[27] A. Coll, R. Ribo, M. Pasenau, E. Escolano, J. Perez, A. Melendo, A. Monros and J. Garate, "GiD: the personal pre and post-processor. User Manual," CIMNE, Technical University of Catalonia, p. $<$ http://gid.cimne.upc.ed>, 2016. 
[28] D. A. Hordijk, "Local approach to fatigue of concrete, PhD Thesis," Delft University of Technology, 1991.

[29] I. Marzec and J. Tejchman, "Enhanced coupled elasto-plastic-damage models to describe concrete behaviour in cyclic laboratory tests: comparison and improvement," Archives of Mechanics, vol. 64, no. 3, pp. 227-259, 2012.

[30] B. Bourdin, G. Francfort and J.-J. Marigo, "Numerical experiments in revisited brittle fracture," Journal of the mechanics and physics of solids, vol. 48, no. 4, pp. 797-826, 2000.

[31] C. Miehe, M. Hofacker and F. Welschinger, "A phase field model for rate-independent crack propagation: robust algorithmic implementation based on operator splits," Computer Methods in Applied Mechanics and Engineering, vol. 199, pp. 2765-2778, 2010.

[32] J.-Y. Wu and V. Nguyen, "A length scale insensitive phase-field damage model for brittle fracture," Journal of the Mechanics and Physics of Solids, vol. 119, pp. 20-42, 2018.

[33] P. Carrara, M. Ambati, R. Alessi and L. De Lorenzis, "A novel framework to model the fatigue behavior of brittle materials based on a variational phase-field approach," Submited to arXiv:1811.02244, 2018. 